BMC

Genomics

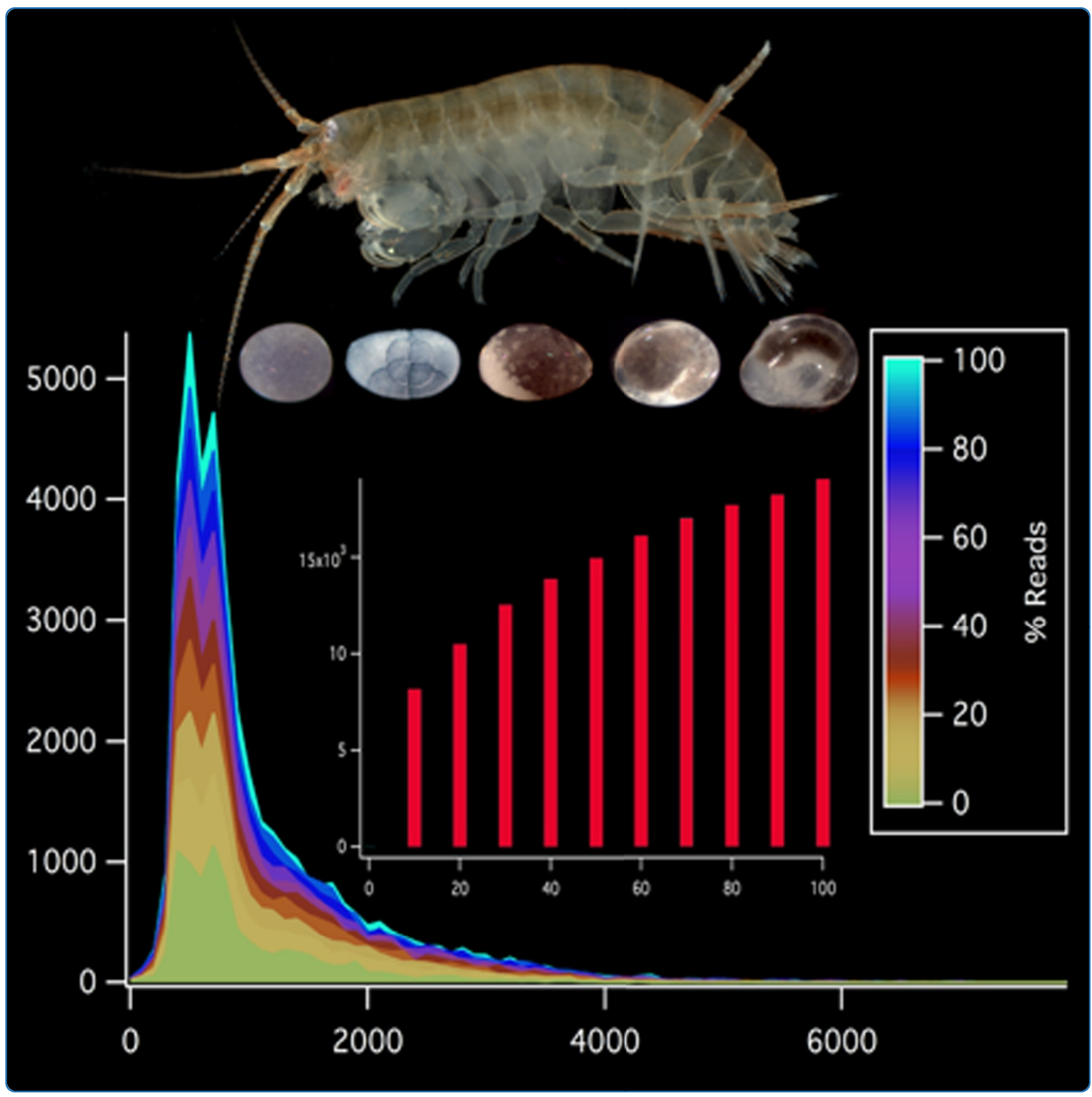

De novo assembly and characterization of a maternal and developmental transcriptome for the emerging model crustacean Parhyale hawaiensis

Zeng et al.

C Biomed Central

Zeng et al. BMC Genomics 2011, 12:581

http://www.biomedcentral.com/1471-2164/12/581 (25 November 2011) 


\title{
De novo assembly and characterization of a maternal and developmental transcriptome for the emerging model crustacean Parhyale hawaiensis
}

Victor Zeng ${ }^{1}$, Karina E Villanueva ${ }^{2}$, Ben S Ewen-Campen ${ }^{1}$, Frederike Alwes ${ }^{1}$, William E Browne ${ }^{2 *}$ and Cassandra G Extavour ${ }^{1 *}$

\begin{abstract}
Background: Arthropods are the most diverse animal phylum, but their genomic resources are relatively few. While the genome of the branchiopod Daphnia pulex is now available, no other large-scale crustacean genomic resources are available for comparison. In particular, genomic resources are lacking for the most tractable laboratory model of crustacean development, the amphipod Parhyale hawaiensis. Insight into shared and divergent characters of crustacean genomes will facilitate interpretation of future developmental, biomedical, and ecological research using crustacean models.

Results: To generate a transcriptome enriched for maternally provided and zygotically transcribed developmental genes, we created cDNA from ovaries and embryos of $P$. hawaiensis. Using 454 pyrosequencing, we sequenced over 1.1 billion bases of this CDNA, and assembled them de novo to create, to our knowledge, the second largest crustacean genomic resource to date. We found an unusually high proportion of $\mathrm{C} 2 \mathrm{H} 2$ zinc finger-containing transcripts, as has also been reported for the genome of the pea aphid Acyrthosiphon pisum. Consistent with previous reports, we detected trans-spliced transcripts, but found that they did not noticeably impact transcriptome assembly. Our assembly products yielded 19,067 unique BLAST hits against $\mathbf{n r}$ (E-value cutoff e-10). These included over 400 predicted transcripts with significant similarity to D. pulex sequences but not to sequences of any other animal. Annotation of several hundred genes revealed $P$. hawaiensis homologues of genes involved in development, gametogenesis, and a majority of the members of six major conserved metazoan signaling pathways.

Conclusions: The amphipod P. hawaiensis has higher transcript complexity than known insect transcriptomes, and trans-splicing does not appear to be a major contributor to this complexity. We discuss the importance of a reliable comparative genomic framework within which to consider findings from new crustacean models such as D. pulex and P. hawaiensis, as well as the need for development of further substantial crustacean genomic resources.
\end{abstract}

\section{Background}

Crustaceans are one of the four major groups that make up the phylum Arthropoda, the most speciose and morphologically diverse animal group [1]. Despite the fact that arthropods as a whole make up the majority of

\footnotetext{
* Correspondence: wbrowne@bio.miami.edu; extavour@oeb.harvard.edu 'Department of Organismic and Evolutionary Biology, Harvard University, 16 Divinity Avenue, Cambridge, MA 02138, USA

2Department of Biology, University of Miami, 234 Cox Science Center, 1301

Memorial Drive, Coral Gables, FL 33146, USA

Full list of author information is available at the end of the article
}

animal species diversity and biomass, until recently the only arthropod represented in the list of NIH model organisms http://www.nih.gov/science/models/ was the fruit fly Drosophila melanogaster. The water flea Daphnia pulex was recently added to this list, and is the only crustacean to date with a publicly accessible sequenced genome [2]. As crustaceans are now widely recognized as sister group to the hexapods [3-7], the phylogenetic position of $D$. pulex suggests that it could serve as a useful outgroup to insects, providing meaningful comparisons with the many insights into developmental and

\section{Biomed Central}

() 2011 Zeng et al; licensee BioMed Central Ltd. This is an Open Access article distributed under the terms of the Creative Commons Attribution License (http://creativecommons.org/licenses/by/2.0), which permits unrestricted use, distribution, and reproduction in any medium, provided the original work is properly cited. 
disease biology provided by work on $D$. melanogaster. However, there are no genomic resources on a scale comparable to the $D$. pulex genome, and so it is still not known to what extent the characteristics of the water flea genome are specific to this animal's ecology or shared by other crustaceans [2]. Moreover, comparisons of biomedically relevant processes and mechanisms between $D$. pulex and other model organisms must be informed by robust phylogenetic hypotheses. At the moment, which specific subgroup of crustaceans is closest to the hexapods (including D. melanogaster) is still a matter of debate [8], but several phylogenetic hypotheses suggest that branchiopods may be more distant from insects than other crustacean groups [6,9-12].

Crustaceans have long been the subject of ecological and evolutionary study, as well as being lucrative commercial species for human consumption. Even for many of the most intensively studied of these [13], surprisingly few genomic projects have been reported. Most of the crustacean EST projects completed to date, notably for the farmed shrimp Litopenaeus vannamei ( 163,000 ESTs) [14-16], the salmon ectoparasite copepod Lepeophtheirus salmonis ( 129,000 ESTs) [17], the porcelain crab Petrolisthes cinctipes ( 98,000 ESTs) [18], and the lobster Homarus americanus ( 52,000 ESTs) [19-21], have all used Sanger sequencing of cDNA libraries. Next generation sequencing technologies are increasingly affordable, accessible and robust even for organisms lacking a sequenced genome [22], but have been reported to be applied to a crustacean de novo transcriptome only once, in the Antarctic krill Euphausia superba [23]. This organism is the subject of ecological and climate change research, but is not a viable laboratory model organism due to its specialized habitat.

While new understanding of developmental and molecular mechanisms in $D$. pulex are expected to follow from its genome sequence, it is important to note that crustaceans have been the subject of comparative embryology for over a century [24], and in recent decades, of evolutionary developmental biology ("evo-devo"). The morphological and molecular mechanistic variations of early embryogenesis, modifications of their body plans and appendage diversifications displayed by crustaceans have all been the subject of studies too numerous to describe here. However, until recently most comparative analyses of crustaceans have been limited to the study of gene expression or experimental embryology, as crustacean models where functional genetic testing is possible are still few in number. The limiting factor for comparative functional experiments is often obtaining specific coding sequences of sufficient length. A large-scale genomic resource for a model crustacean would therefore greatly facilitate development and deployment of transgenic tools.
The amphipod Parhyale hawaiensis has emerged as an important laboratory model crustacean species over the last several years [25]. P. hawaiensis was first described in the Hawaiian islands [24], but it occupies intertidal marine habitats worldwide. Laboratory husbandry is easy and affordable, and inbred lab cultures produce hundreds of embryos year-round, providing ample material for developmental studies. Fate map and cell lineage analyses of the early embryo show that all three germ layers and the germ line are determined by the eight cell stage [26], and clonal populations show predictable patterns at least up until gastrulation [27]. Despite this apparently "mosaic" embryonic development, significant regulative properties have also been described for the embryonic mesoderm and ectoderm [28]. Molecular techniques for the study of development, including stable transgenesis [29-31] and gene knockdown [32-34] are arguably better established for $P$. hawaiensis than for any other laboratory crustacean model.

However, the number of $P$. hawaiensis developmental genes available as GenBank accessions is less than 25. Progress in understanding the development of $P$. hawaiensis is thus is limited by the relative paucity of publicly available cloned coding and regulatory regions. Indeed, since the development of germ line transgenesis in $P$. hawaiensis, its use in developmental studies has been reported only three times $[30,31,34]$.

Analysis of phenotypes of genomic transgene insertions [29] and case studies of intron sizes [35] are consistent with an extremely large genome size for this amphipod. Accordingly, the genome size of $P$. hawaiensis is estimated to be $2.98 \mathrm{~Gb}$ ([35], R. Gregory and C. Extavour, unpublished), very near that of Homo sapiens [36,37]. A genomic BAC library for $P$. hawaiensis has been created and partially sequenced [35] but is not yet available in GenBank. The genome of the closely related amphipod Jassa marmorata, with a much smaller genome of $690 \mathrm{Mbp}(\sim 4$ times the size of the $D$. melanogaster genome) has been approved for whole genome, BAC end, and EST sequencing by the Joint Genome Institute [13], but this work is still ongoing, and laboratory culture of this amphipod is difficult. A large transcriptome dataset for P. hawaiensis would thus be a highly valuable resource for several scientific communities, and would in addition assist with the annotation of planned amphipod genomic projects. Recent construction of a de novo transcriptome for the milkweed bug Oncopeltus fasciatus, which also lacks a sequenced genome [22], suggested that 454 pyrosequencing would be a fruitful approach to obtaining a large scale $P$. hawaiensis transcriptome.

Here we present the de novo assembly of a maternal and embryonic transcriptome for $P$. hawaiensis, sequenced with 454 Titanium pyrosequencing. We describe particular features of the $P$. hawaiensis transcriptome that were 
revealed during assembly and annotation, including the presence of trans-splicing and enrichment for $\mathrm{C} 2 \mathrm{H} 2 \mathrm{Zn}$ finger domain-containing transcripts. We annotate orthologues of genes involved in several major developmental patterning processes, gametogenesis in males and females, and members of major conserved animal signaling pathways. We observe a high proportion of apparently unique sequences in the transcriptome, and discuss these findings in the light of observations on the D. pulex genome and other existing crustacean genomic resources.

\section{Results and Discussion}

\section{Collection and preparation of material for ovarian and} embryonic cDNA libraries

Our goal was to create a transcriptome containing genes relevant to embryonic development, including both maternally provided and zygotically transcribed genes. All three germ layers and the germ line are determined by the eight cell stage [26], and we wished to capture transcripts from that early stage (Figure 1D top). We also wished to sample the intensively studied germ band stage (Figure 1D bottom), when major body axes have been patterned and trunk segmentation is ongoing [38], as well as later stages (S20-S27) when organogenesis is predominant. We therefore collected ovaries (Figure 1C) and embryos from all stages of embryogenesis (Figure 1E), extracted mRNA and prepared cDNA for 454 pyrosequencing. Because early stage embryos have many fewer cells than later stage embryos, we anticipated that transcripts present in early embryos might suffer low representation if our collection contained equal numbers of embryos of all stages. We therefore collected greater numbers of earlier stages than of later stages (Additional File 1).

\section{Sequencing and assembly of the $P$. hawaiensis transcriptome}

We sequenced a total of 3,172,925 reads (Table 1) with a median read length of 400 bp (Figure 2A; Additional File
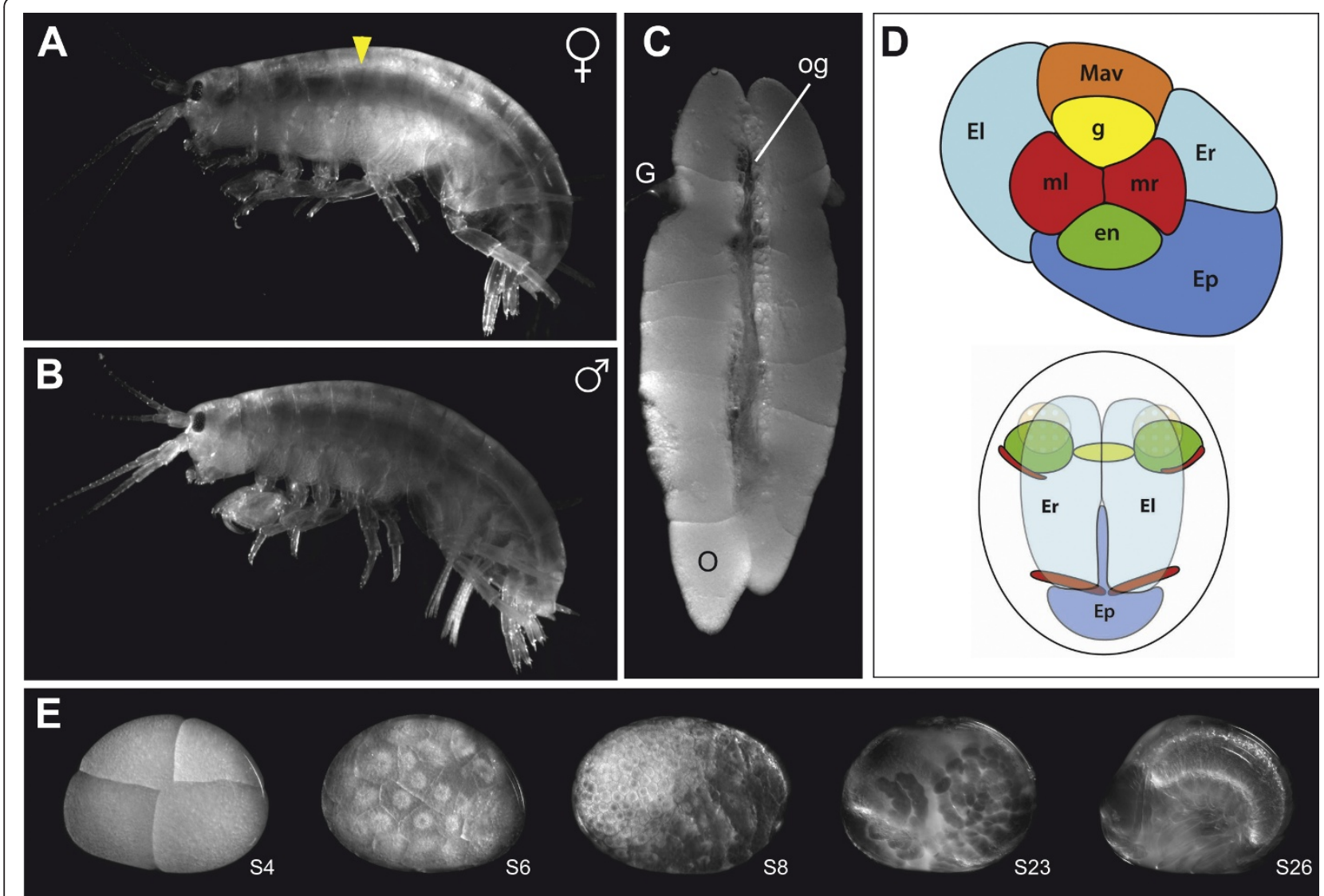

Figure 1 Parhyale hawaiensis and the tissues used to construct a de novo transcriptome. (A) Adult female amphipod, P. hawaiensis. (B) Adult male. (C) Ovaries of adult female. Oocytes and oogonia are visible at various stages of growth. (D) Schematic drawings of the eight cell stage (top), at which all germ layers and the germ line are specified, and the germ band stage (bottom). Both of these signature stages are represented in this transcriptome. $(E)$ A sample of the range of stages of $P$. hawaiensis embryogenesis represented in this transcriptome; stages as per [55]. Embryos from as early as S1 (one cell stage) and as late as S27 (just before hatching) were sampled; see Additional File 1 for details. G: gonoduct; O: late stage oocyte; og: younger oocytes and oogonia. Anterior is to the left in $A, B$, and $E$, and up in $C$. 
Table $1 P$. hawaiensis transcriptome assembly statistics

\begin{tabular}{lc}
\hline Raw reads (base pairs) & $\mathbf{3 , 1 7 2 , 9 2 5}(\mathbf{1 , 1 7 9 , 5 4 4 , 2 9 1 )}$ \\
\hline Assembled reads & $3,157,373$ \\
Isotigs & 35,301 \\
Isotig N50 & 1,510 \\
Singletons (\% of assembled reads) & $276,564(8.8 \%)$ \\
\# Unique BLAST hits & 19,067 \\
Isogroups ("genes") & 25,735 \\
Mean \# isotigs per isogroup & 1.4 \\
Newbler Contigs ("exons") & 89,664 \\
Mean \# contigs per isotig & 2.1 \\
\hline
\end{tabular}

2). For assembly of the de novo transcriptome we used Newbler v2.5 (Roche). Newbler's terminology for assembled reads distinguishes between contigs (groups of assembled reads with overlapping regions considered significant, i.e. putative exons), isotigs (continuous paths through a set of contigs, i.e. putative transcripts), and isogroups (groups of isotigs assembled from the same set of contigs, i.e. putative genes). For continuity, we use this terminology throughout this paper to refer to the products of the Newbler assembly.

The default parameters of Newbler v2.5 were used to screen for adapters and eliminate poor quality reads. The resulting $3,157,373$ reads ( $99.5 \%$ of raw reads) were then assembled (Table 1). 2,349,266 (74.4\%) of the screened reads were incorporated into assembled sequences (isotigs or contigs), with 276,564 (8.8\%) singletons remaining. 531,543 reads ( $16.8 \%$ of reads subjected to assembly) were excluded because they were only partially assembled
(431,372; $13.7 \%)$, from repeat regions $(5,022 ; 0.2 \%)$, outliers $(86,822 ; 2.7 \%)$, or too short (< 40 bp: 8,327 ; $0.3 \%)$. The assembly contained 89,664 contigs, which grouped into 35,301 isotigs. 18,615 (52.7\%) of these isotigs were made up of only one contig, and the average number of contigs per isotigs was 2.1. The isotig N50 length was $1,510 \mathrm{bp}$, and the number of isogroups was $25,735(18,565$ (72.1\%) of these comprised only one isotig, and the mean number of isotigs per isogroup was 1.4). The average coverage per contig was 7.1 reads/bp (Additional File 3). Averaging across all bases in the entire assembly, the average coverage per base pair was 25.4, meaning that every base pair in the transcriptome was sequenced 25.4 times on average. This coverage is high compared to typical numbers for de novo transcriptome assemblies, and should be helpful for distinguishing SNPs and indels from potential sequencing errors in raw reads [39]. Fasta files of all assembly products are freely available from the authors.

\section{BLAST mapping of non-redundant transcriptome sequences}

Using an E-value cutoff of 1e-10, we first used BLASTX to map all non-redundant assembly sequences to $\mathbf{n r}$ (total number of sequences $=311,865=35,301$ isotigs $+276,564$ singletons), and obtained a total of 20,007 BLAST hits (Table 2). Of the 35,301 isotigs, 10,424 (29.5\%) had at least one hit (including 9,715 contigs (10.8\%), and of the 276,564 singletons, 9,583 (3.5\%) had hits. The majority of these BLAST hits were unique: among the isotigs there were 10,203 (28.9\%) unique hits, and among the singletons
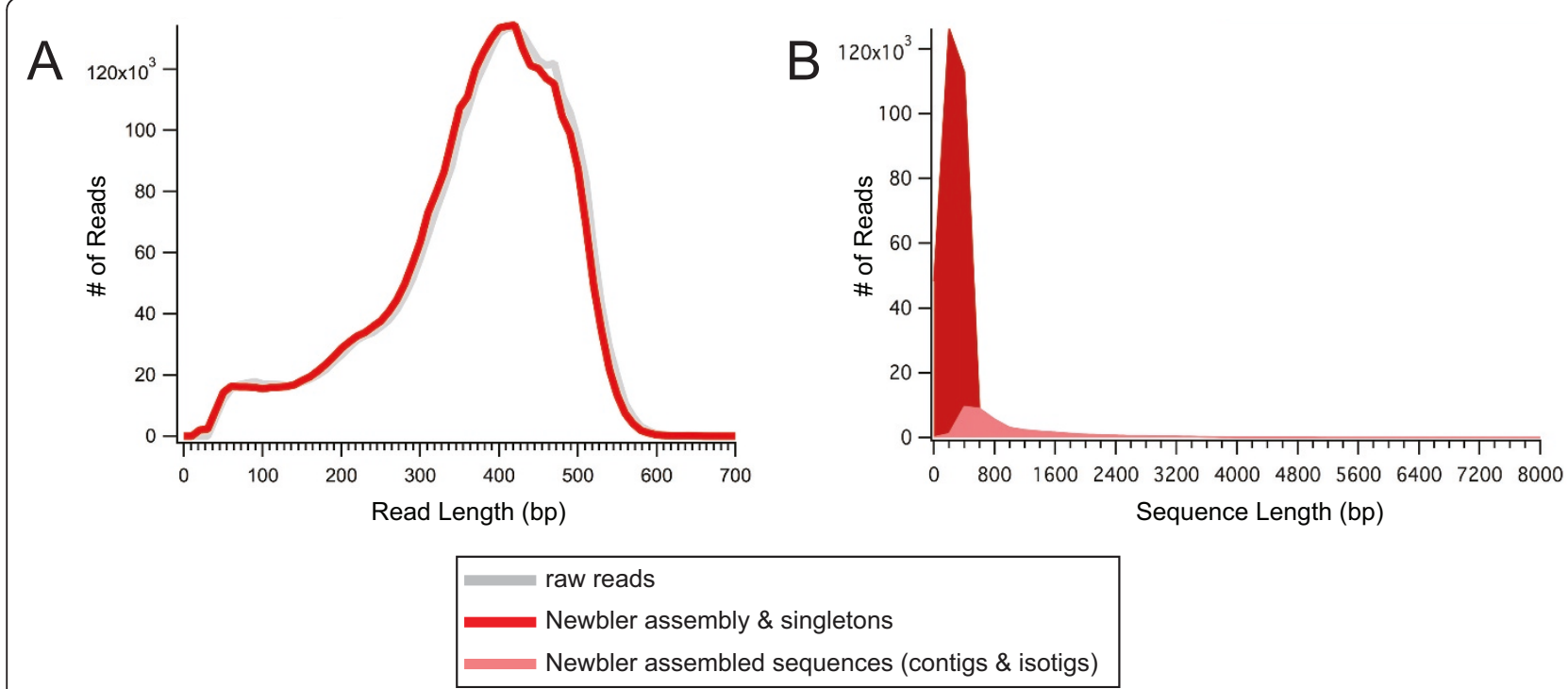

Figure 2 Read lengths of raw, trimmed, and assembled reads. (A) The raw reads (grey) ranged in length from < 40 bp to 1196 bp, with a mean read length of $400 \mathrm{bp}$. The distribution of read lengths of those reads chosen for assembly (red) was comparable to that of raw reads for the Newbler assembly (grey). (B) Read length distributions from all products of assembly of trimmed reads. The longest isotig per isogroup is shown. Removing singletons (unassembled reads) from these data shows that most assembly products under $\sim 600$ bp are singletons, i.e. that the vast majority of assembly products are transcript models over $600 \mathrm{bp}$ (pink). 
Table 2 P. hawaiensis transcriptome BLAST results

\begin{tabular}{|c|c|c|c|}
\hline & $\begin{array}{c}\# \\
\text { Sequences }\end{array}$ & $\begin{array}{l}\text { \# BLAST hits against } \mathrm{nr}^{1} \\
(\%)\end{array}$ & $\begin{array}{c}\text { \# Unique BLAST hits against } \mathrm{nr}^{1} \\
(\%)\end{array}$ \\
\hline Isogroups & 25,735 & $n / a^{2}$ & $n / a^{2}$ \\
\hline Isotigs & 35,301 & $10,424(29.5 \%)$ & $10,203(28.9 \%)$ \\
\hline Contigs & 89,664 & $9,715(10.8 \%)$ & $n / a^{3}$ \\
\hline Singletons & 276,564 & $9,583(3.5 \%)$ & $8,864(3.2 \%)$ \\
\hline $\begin{array}{l}\text { Total Non-Redundant Assembly Sequences (= isotigs + } \\
\text { singletons) }\end{array}$ & 311,865 & $20,007(6.4 \%)$ & $19,067(6.1 \%)$ \\
\hline
\end{tabular}

there were 8,864 (3.2\%) unique hits (Table 2; see 'Sequence Annotation' within Materials and Methods for our determination of "unique BLAST hits"). In summary, among all non-redundant assembly sequences (i.e. isotigs + singletons), we obtained 19,067 unique BLAST hits (Table 2). These BLAST results may mean that the transcriptome contains as many as 19,067 unique gene transcripts. However, as for all de novo assemblies, several caveats must be considered. First, different regions of a single transcript may have different best top BLAST hits. Our assembly likely contains sequences that belong to the same transcript but are too far apart to be assembled together, and so would be considered "different genes." As a result, the number of unique BLAST hits may be an overestimate of unique gene number (see also discussion of total gene number estimation in section "Comparison with other arthropod genomic resources" below). Second, 24,877 isotigs (70.5\% of all isotigs) and 266,981 singletons (96.5\% of all singletons) did not yield BLAST hits that met our E-value cutoff of e-10. These values are comparable to or higher than those obtained in other de novo transcriptome analyses [40-45]. However, these unmatched sequences may represent transcript fragments whose similarities to known genes is too poor to meet our E-value cutoff, or are noncoding. It is therefore not formally possible from de novo assembly to know whether the 19,067 unique BLAST hit number over- or under-estimates the true number of genes contained in our transcriptome.

\section{Transcriptome Gene Ontology (GO) term annotation}

We used Blast2GO [46] to obtain the gene ontology terms associated with the top 50 BLAST hits for each nonredundant assembly sequence. Of the 19,067 sequences with unique BLAST hits as per our 1e-10 E-value cutoff, 9,451 (49.6\%) of these had GO terms associated with them. To determine whether or not major categories of genes were missing or underrepresented in our transcriptome, we compared the proportions of sequences in selected GO term groups to the proportions in these categories observed in the predicted transcript complement of the only crustacean with a sequenced genome, $D$. pulex (see Materials and methods; Figure 3). We did not find significant differences in the proportion of genes in the examined GO term categories between the P. hawaiensis transcriptome and the water flea genome, suggesting that our transcriptome does not lack major functional categories of genes. Interestingly, not only were the proportions similar for the two crustaceans, they also closely matched the profiles observed for the sequenced genome of the insect D. melanogaster, and a de novo insect transcriptome from a milkweed bug [22] (Figure 3), suggesting that arthropod species as widely diverged as fruit flies and water fleas share similar proportional gene expression profiles in certain functional genetic categories.

\section{Unusual characteristics of the $P$. hawaiensis transcriptome}

$P$. hawaiensis has been found to employ trans-splicing among its genetic regulatory mechanisms [47]. In this mechanism, sections of transcripts transcribed from independent genomic loci are spliced together post-transcriptionally to form a novel transcript. Such trans-spliced transcripts are recognizable because they contain a diagnostic splice-leader sequence. We wished to determine to what extent this additional transcript complexity affected our assembly and our ability to assign high-confidence BLAST annotations. We therefore processed the trimmed, preassembly reads to remove those containing the diagnostic splice-leader sequences $(2,584 ; 0.1 \%)$, and performed a Newbler v2.5 assembly on the remaining reads. The results of this assembly were not noticeably different from the complete assembly, nor did the number of unique BLAST hits increase (Additional File 4). We therefore concluded that the presence of trans-spliced transcripts did not significantly affect our transcriptome assembly or annotation.

When we considered the species identity of the top BLAST hit for each isotig and singleton (see Methods), we found that a high proportion (50.5\%) of our assembly sequences most closely matched sequences from other arthropods (Additional File 5). However, an unexpectedly high proportion (12.2\%) was from the lancelet 


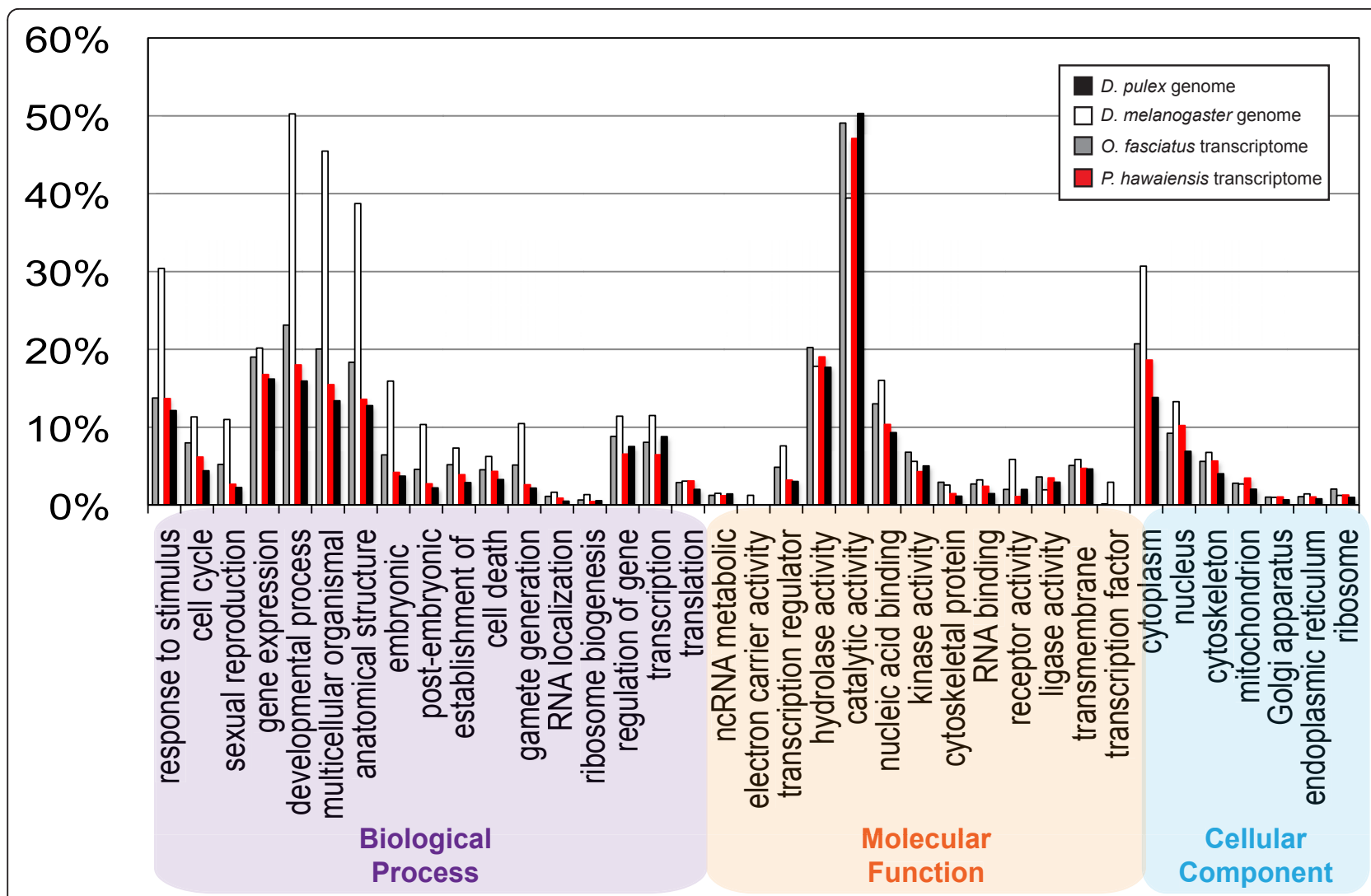

Figure $3 \mathrm{GO}$ term distribution of BLAST hits from the $P$. hawaiensis transcriptome. For comparison, GO term distributions of transcript predictions from a crustacean (Daphnia pulex: water flea) and an insect (Drosophila melanogaster: fruit fly) with sequenced genomes are shown, as well as those from the largest available de novo pyrosequenced insect transcriptome to date (Oncopeltus fasciatus: milkweed bug [22]). Selected GO categories are shown within the top-level divisions of Biological Process, Molecular Function, and Cellular Component. Column heights reflect the percentage of annotated sequences that mapped to a given Biological Process $G O$ term.

Branchiostoma floridae, which is phylogenetically very far removed from $P$. hawaiensis. When we examined those sequences with a lancelet sequence as top hit, we noticed that (a) for many of them, the top 50 BLAST hits were all B. floridae; and (b) the majority of them seemed to contain $\mathrm{C} 2 \mathrm{H} 2$ zinc fingers with a specific linker sequence (TGEKP) between $\mathrm{C} 2 \mathrm{H} 2$ domains.

The genome of at least one other arthropod, the pea aphid Acyrthosiphon pisum, has been found to be unusually rich in genes containing the same $\mathrm{C} 2 \mathrm{H} 2$ zinc finger and diagnostic linker sequence [48]. We reasoned that if both the amphioxus [49] and amphipod genomes happened to contain a high proportion of such genes, this could be responsible for the apparent high similarity of several transcripts in these two species. To test this, we removed reads containing $\mathrm{C} 2 \mathrm{H} 2$ zinc finger-encoding sequences, following a previously defined low-stringency definition of a $\mathrm{C} 2 \mathrm{H} 2$ zinc finger domain [50]. We then assembled the remaining reads with Newbler v2.5, and again scanned for and removed contigs and isotigs containing the motif. The remaining assembly products were mapped against $\mathbf{n r}$ using BLASTX, and the species identities of their top hits were compared with those obtained from the complete assembly. We found that the new assembly retained a large proportion $(54.3 \%)$ of arthropod hits, but that the number of $B$. floridae hits had dropped to $4.3 \%$, and was now comparable to the proportion of hits obtained from other deuterostome phyla (Figure 4, Additional File 5).

\section{Comparison with other arthropod genomic resources}

The BLAST hit rate of the P. hawaiensis isotigs was $29.5 \%$. This is higher than those reported in other de novo transcriptome analyses [40-45], including that of an arctic crustacean [23], but much lower than the $43.4 \%$ obtained from a previously analyzed de novo insect maternal/ embryonic transcriptome [22]. The $~ 70 \%$ of $P$. hawaiensis isotigs without a high confidence BLAST hit could be either $P$. hawaiensis-specific genes, or genes with relatively lower similarity to known genes. In addition, it is possible that many of our transcripts represent untranslated sequences rather than coding regions (for example, UTRs 


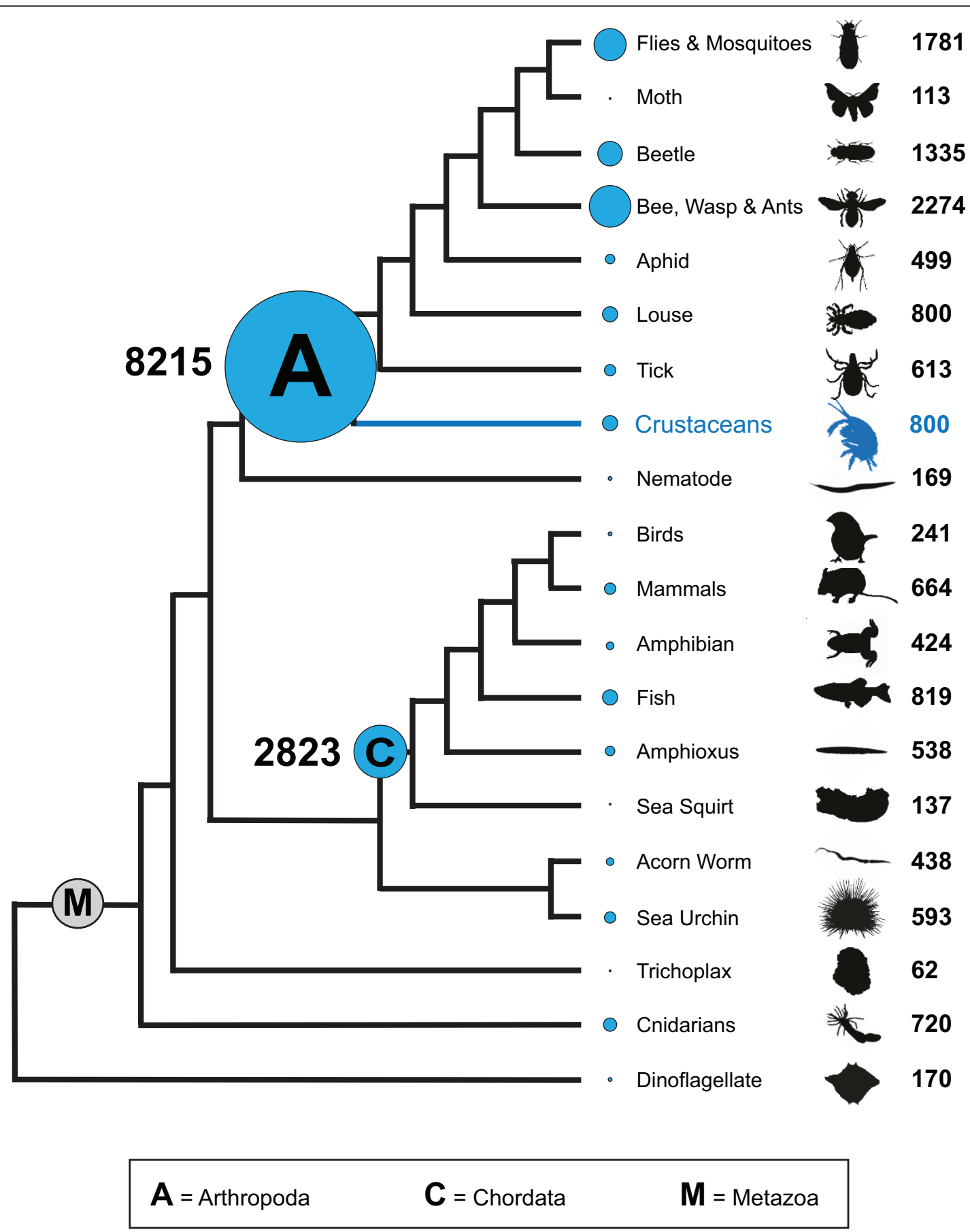

Figure 4 Phylogenetic distribution of species of top unique BLAST hits for the $P$. hawaiensis transcriptome sequences. First, raw reads that contained $\mathrm{C} 2 \mathrm{H} 2$ zinc finger sequences were removed before assembly. Second, assembled sequences that contained $\mathrm{C} 2 \mathrm{H} 2 \mathrm{zinc}$ finger sequences were removed from the output file. We then determined the unique BLAST hits for the remaining non-redundant assembly products (isotigs + singletons). $90 \%$ of the resulting unique BLAST hits were from species belonging to the clades shown. Blue circles are scaled according to the proportion of sequences with species belonging the clade indicated as their top BLAST hit. Over 50\% of top BLAST hits are from arthropod species. Phylogenetic tree modified from $[63,74,75]$. Hits from the following most abundant species are represented: D. mojavensis, D. willistoni, D. ananassae, D. grimshawi, D. pseudoobscura pseudoobscura, Aedes aegypti, Anopheles gambiae, Culex quinquefasciatus (Flies \& Mosquitoes), Bombyx mori (Moth), Tribolium castaneum (Beetle), Harpegnathos saltator, Camponotus floridanus, Apis mellifera, Nasonia vitripennnis (Bee, Wasp \& Ants), Acyrthosiphon pisum (Aphid), Pediculus humanus corporis (Louse), Ixodes scapularis (Tick), Penaeus monodon, Lepeophtheirus salmonis, Litopenaeus vannamei (Crustaceans), Caenorhabditis remanei (Nematode), Gallus gallus, Taeniopygia guttata (Birds), Rattus norvegicus, Mus musculus, Monodelphis domestica (Mammals), Xenopus laevis, X. tropicalis (Amphibian), Danio rerio, Tetraodon nigroviridis (Fish), Branchiostoma floridae (Amphioxus), Ciona intestinalis (Sea Squirt), Saccoglossus kowalevskii (Acorn Worm), Strongylocentrotus purpuratus (Sea Urchin), Trichoplax adherens (Trichoplax), Hydra magnipapillata, Nematostella vectensis (Cnidarians), Perkinsus marinus (Dinoflagellate). See Additional File 5 for details. 
or noncoding RNAs), and therefore do not match $\mathbf{n r}$ protein sequences.

The relatively low proportion of hits may also reflect the fact that until recently (and including the version of $\mathbf{n r}$ we used for our analysis, which did not include the D. pulex genome at the time of analysis) very few crustacean sequences were included in $\mathbf{n r}$. The $D$. pulex genome also contains a high proportion ( 36\%) of apparently Daphniaspecific genes, thought to be largely the result of amplification of selected gene families [2]. Noting that the E-value cutoff used for the D. pulex analyses (1e-5) was slightly more relaxed than that used in our initial analysis (1e-10), we repeated the BLASTX of our non-redundant $P$. hawaiensis transcriptome sequences against $\mathbf{n r}$ with an E-value cutoff of 1e-5. This yielded 26,494 unique BLAST hits (including $34.8 \%$ of isotigs and $5.7 \%$ of singletons). We therefore found that even adjusting the E-value cutoff to that used for characterization of the $D$. pulex genome, $\sim 59 \%$ of the sequences in this $P$. hawaiensis transcriptome lack significant similarity to other characterized animal sequences. These observations suggest that crustaceans may have more species- or clade-specific genes than previously appreciated. Alternatively, these high numbers of apparently lineage-restricted genes may simply reflect the paucity of crustacean genomic resources currently in public databases.

Those $P$. hawaiensis transcriptome sequences which failed to obtain a significant BLAST hit when compared with nr might share more similarity with other crustaceans. To test this, we used BLAST to compare all $P$. hawaiensis transcriptome sequences that had failed our 1e-10 E-value cutoff against $\mathbf{n r}$, with the predicted D. pulex transcriptome (see Methods) using BLASTX and a 1e-10 E-value cutoff. We then used BLAST to compare the obtained sequences with $\mathbf{n r}$ to determine their putative identities. We did not set an E-value limit for this second BLAST, in order to recover at least some minimal information about the identity of these genes (E-values for a subset of these, described below, are shown in Additional File 6). We found that $47.9 \%$ of these sequences came from arthropods, only $2.5 \%$ of which were crustaceans. The low crustacean representation in $\mathbf{n r}$ makes it difficult to obtain high confidence BLAST hits for this group. We therefore focused on determining what proportion of "nr orphan" $P$. hawaiensis sequences were highly similar to sequences from $D$. pulex by comparing the E-values for BLAST hits against both $\mathbf{n r}$ and against the $D$. pulex predicted transcriptome. We found that of the 423 $P$. hawaiensis sequences with higher similarity to $D$. pulex genes than to anything in nr (Additional File 6), 381 (90.1\%) of these had E-values at least an order of magnitude higher for $D$. pulex compared to $\mathbf{n r}$, and 30 of these (7.1\% of total) had E-values greater than one for $\mathbf{n r}$ hits, but $D$. pulex hit E-values of $1 \mathrm{e}-11$ or lower. Most of this
"Daphnia-like" group matched arthropod sequences that were previously annotated as "hypothetical proteins," suggesting that non-insect crustacean sequence annotation could improve future annotation of the existing insect genomes.

The D. pulex genome has been found to contain a high number of genes (at least 30,907) [2]. Without a genome sequence for $P$. hawaiensis, we cannot accurately estimate gene number in order to perform a rigorous comparison with $D$. pulex. However, our transcriptome assembly identified 25,735 isogroups. Because isogroups are groups of isotigs assembled from the same set of contigs, isogroups may represent putative genes, with each isotig of the isogroup representing a transcript variant, for example a splice variant. We therefore speculate that $P$. hawaiensis, with a genome over one order of magnitude larger than that of D. pulex (C. Extavour \& R. Gregory, unpublished), may also have a high gene number of at least 25,735 genes.

However, using isogroup number of this de novo transcriptome as a proxy for total gene number has two significant limitations. The first is the result of our chosen tissue sampling strategy: this transcriptome does not capture postembryonic gene expression. After hatching, expression of several genes with exclusively juvenile or adult roles is likely, including at minimum additional genes associated with molting, behavior, and gametogenesis. It is therefore possible that the number of isogroups in our assembly underestimates the true gene number in P. hawaiensis.

The second limitation is the unavoidable result of any de novo assembly, which is that if two sequences from the same transcript do not share significant overlap, they will appear as separate assembly products rather than as a single transcript (see also discussion of unique BLAST hit number in section "BLAST mapping of non-redundant transcriptome sequences" above). This could result in the number of isogroups being an overestimate of the true number of genes. A further complication results from Newbler v2.5's method of handling isogroups made of multiple isotigs. When we performed our assembly, we limited the number of isotigs in one isogroup to 10 ("-it" flag; see Methods). This has the advantage of avoiding isogroups composed of large numbers of isotigs, as we suspect that in P. hawaiensis, as shown for other animals, the vast majority of genes have fewer than ten splicing isoforms [51]. However, it can also result in problematic isogroup number calculation, because isogroups that exceed the number of isotigs per isogroup threshold are returned to the assembly file as contigs rather than isotigs, thus inflating the gene number estimate. In summary, in order to determine whether high numbers of species- or cladespecific genes is a general characteristic of crustaceans, and the true extent of species-specific genes for $P$. hawaiensis, more deep genomic resources will have to 
be developed for this amphipod and for more crustaceans of diverse classes.

The high gene number of $D$. pulex is thought to be due to lineage-specific expansions of gene families [2]. These expansions may play adaptive roles in the water flea's ecology [2], or they may be a general feature of crustacean genomes that was previously unappreciated due to the paucity of crustacean genomic resources. It is therefore of interest to determine the extent of gene family expansion in P. hawaiensis. In order for our de novo transcriptome to provide a rigorous answer to this question, we would need to distinguish between transcripts of paralogues, and sequences originating from the same transcript that do not overlap enough to belong to the same isotig, or even obtain the same set of top BLAST hits. This distinction is not unambiguously possible, given the absence of a reference genome. However, we performed a preliminary analysis of putative gene expansion in $P$. hawaiensis, focusing on those gene families found to be expanded in the D. pulex genome. Our analysis conservatively included only those $P$. hawaiensis isotigs that had a top BLAST hit against a duplicated D. pulex gene. We also made sure that these isotigs had the same set of top BLAST hits but belonged to different isogroups, and were therefore likely to represent paralogues rather than splice variants (see Methods). We found that in general, highly expanded D. pulex gene families had more putative paralogues (isogroups) in P. hawaiensis, relative to less expanded gene families (Figure 5). It is therefore possible that gene family expansions are a common feature of certain enzymatic gene families in some crustaceans, although genome sequencing will ultimately be needed to provide definitive answers to this question.

\section{Assessment of depth and transcript coverage of the transcriptome}

Although the 19,067 unique BLAST hits that we identified may represent unique genes, as discussed extensively above we cannot verify how many transcripts are encoded by the genome in the absence of an annotated genome

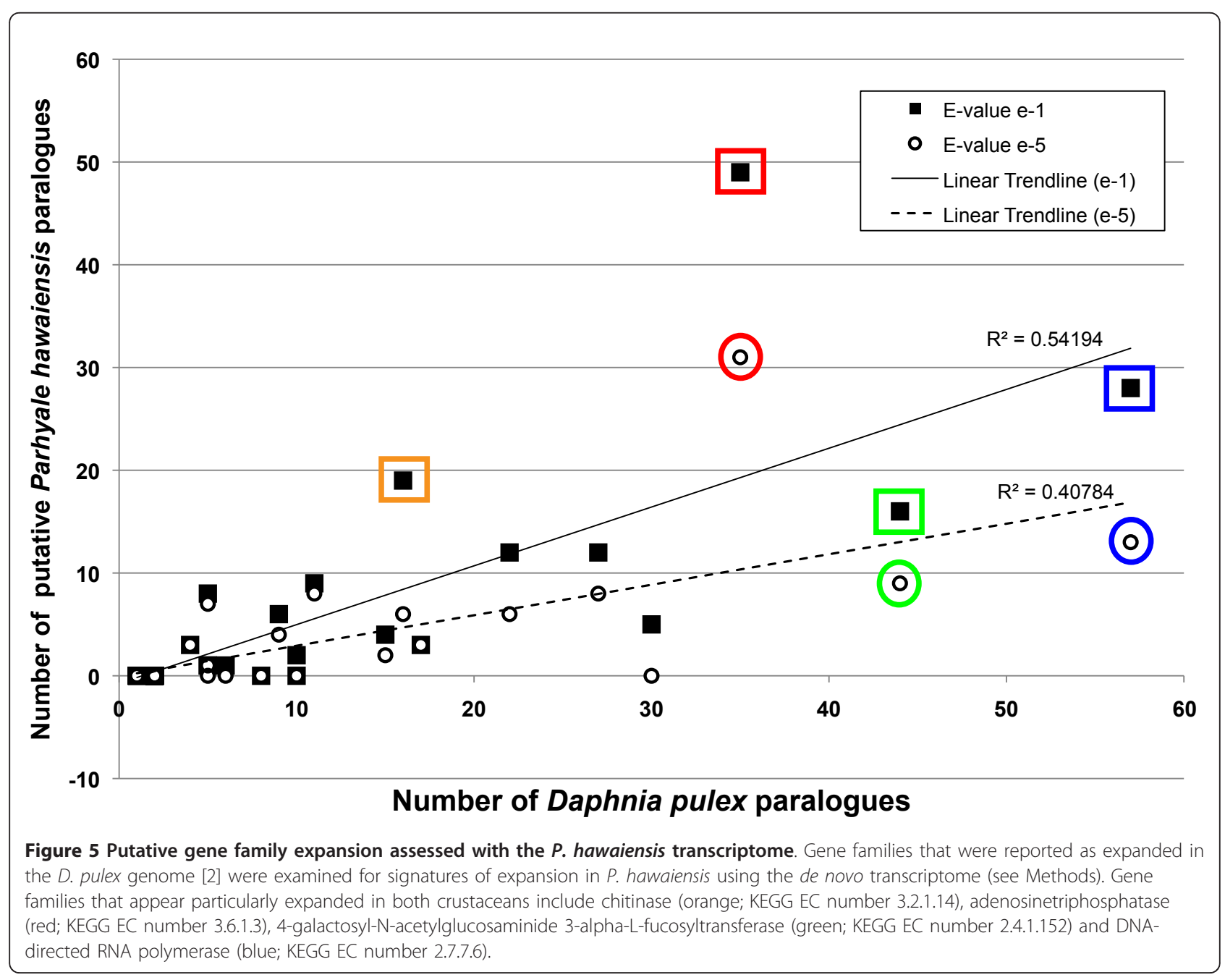


sequence. However, we wished to estimate how deeply we had sequenced those transcripts that were present in our oogenesis/embryogenesis cDNA sample. To do this, we assembled progressively larger random subsets of the total reads. For each subassembly, we used BLAST to compare our non-redundant transcriptome sequences with $\mathbf{n r}$, and assessed the number of unique BLAST hits to see to what extent adding sequence data from the same sample improved gene discovery. Even with the maximum number of over 3 million reads, we did not observe a plateau in the gene discovery rate (Figure 6A). This suggests that despite the considerable depth of our coverage, sequencing more reads from the same sample could yield even more new gene discovery. This is in contrast with a recently constructed de novo maternal and embryonic transcriptome for an insect, which was comprised of only 2 million reads and yet saturated gene discovery in the cDNA sample that was sequenced [22]. The increased complexity of the amphipod transcriptome may reflect its large genome size ([35], C. Extavour and R. Gregory, unpublished), high putative gene number (this study), or large predicted intron sizes [35].

Total isotig length increased steadily as progressively larger subsets of reads were assembled (Figure 6B). While small numbers of isotigs over 4,500 bp could be obtained with as few as $\sim 300,000$ reads, robust recovery of isotigs longer than 6,500 bp required assembly of at least $60 \%$ of our total reads, or $\sim 1.9$ million reads. This demonstrates that increased depth of sequencing, in addition to improving gene discovery, has the added benefit of increasing predicted transcript lengths, thereby facilitating their annotation and making them more immediately useful for downstream applications. In a related analysis, we searched the transcriptome for the presence of the small number of $P$. hawaiensis developmental genes available as GenBank accessions, and found that 52.4\%
$(11 / 21)$ were present (Additional File 7). However, only for one of these genes (Ph-prospero) did our transcriptome add sequence data to the GenBank accession (Additional File 8). This may be a reflection of both the relatively rarity of these transcripts, and the fact that those genes identified to date have been the subject of intense developmental studies, and so sequences of considerable length have already been cloned to close to full length [30,34,52-57].

The assembly yielded isotigs as long as 7,936 bp, with average length $1,128 \mathrm{bp}$ (Figure 6B, C). However, we wished to determine what fraction of true full transcript length was likely to be contained by these isotigs. To do this, we used the methods of O'Neil and colleagues [39] in calculating the ortholog hit ratio for isotigs, contigs, and singletons. We found that $60.2 \%$ of isotigs represented over $50 \%$ of putative true full-length transcripts compared with predicted $D$. pulex transcripts, and $35.0 \%$ of isotigs were over $80 \%$ full length (Figure 7A). These ratios were not significantly higher than those obtained by comparing transcriptome sequences to $D$. melanogaster transcripts (58.1\% and $33.2 \%$ respectively), suggesting that $P$. hawaiensis sequences have comparable similarity to those of the water flea and the fruit fly. Further, comparing transcripts from the fully sequenced genomes of $D$. pulex and D. melanogaster yields ortholog hit ratio values of $65.1 \%$ (above 0.5 ) and $41.7 \%$ (above 0.8 ) respectively, which are similar to the $P$. hawaiensis/D. pulex comparison values. These values are consistent with the increasing support for hypotheses of crustacean paraphyly, which predict large divergences between all of the lineages leading to these arthropod species [see for example 6]. The current state of understanding of crustacean-hexapod phylogenetic relationships (see Conclusions) therefore does not allow straightforward predictions of which pair of these three transcriptomes should be most similar to each other.
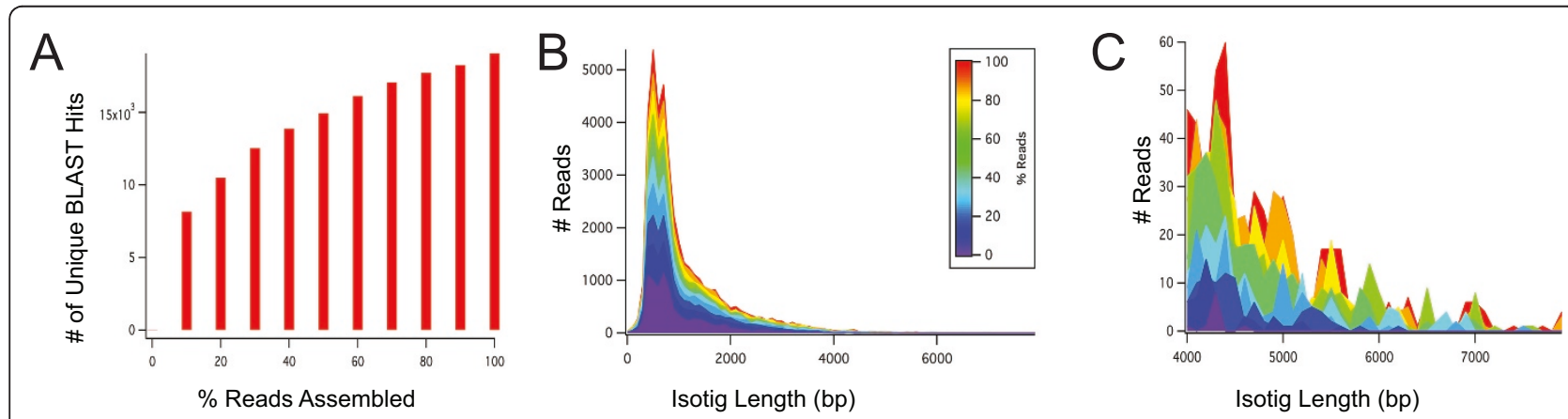

Figure 6 Assessing complexity and depth of the $P$. hawaiensis transcriptome. Randomly sampled subsets of increasing percentages of the total number of reads, in increments of $10 \%$, were used to generate progressively larger sub-assemblies using Newbler v2.5. (A) The number of unique BLAST hits (performed against $\mathbf{n r}$ ) continues to increase as more sequences from this sample are assembled. (B) Full range of isotig length distribution for each sub-assembly. The isotig length distribution remains similar across all sub-assemblies for the shorter $(<5000$ bp) reads in the assembly. (C) High read length ( $>4000$ bp) range of assembled read length distribution for each sub-assembly Assembly of reads with length $\geq 5000$ bp requires assembly of at least $\approx 60 \%$ of our reads, or $\approx 1.9$ million reads. Legend shown in $B$ applies to both $B$ and $C$. 


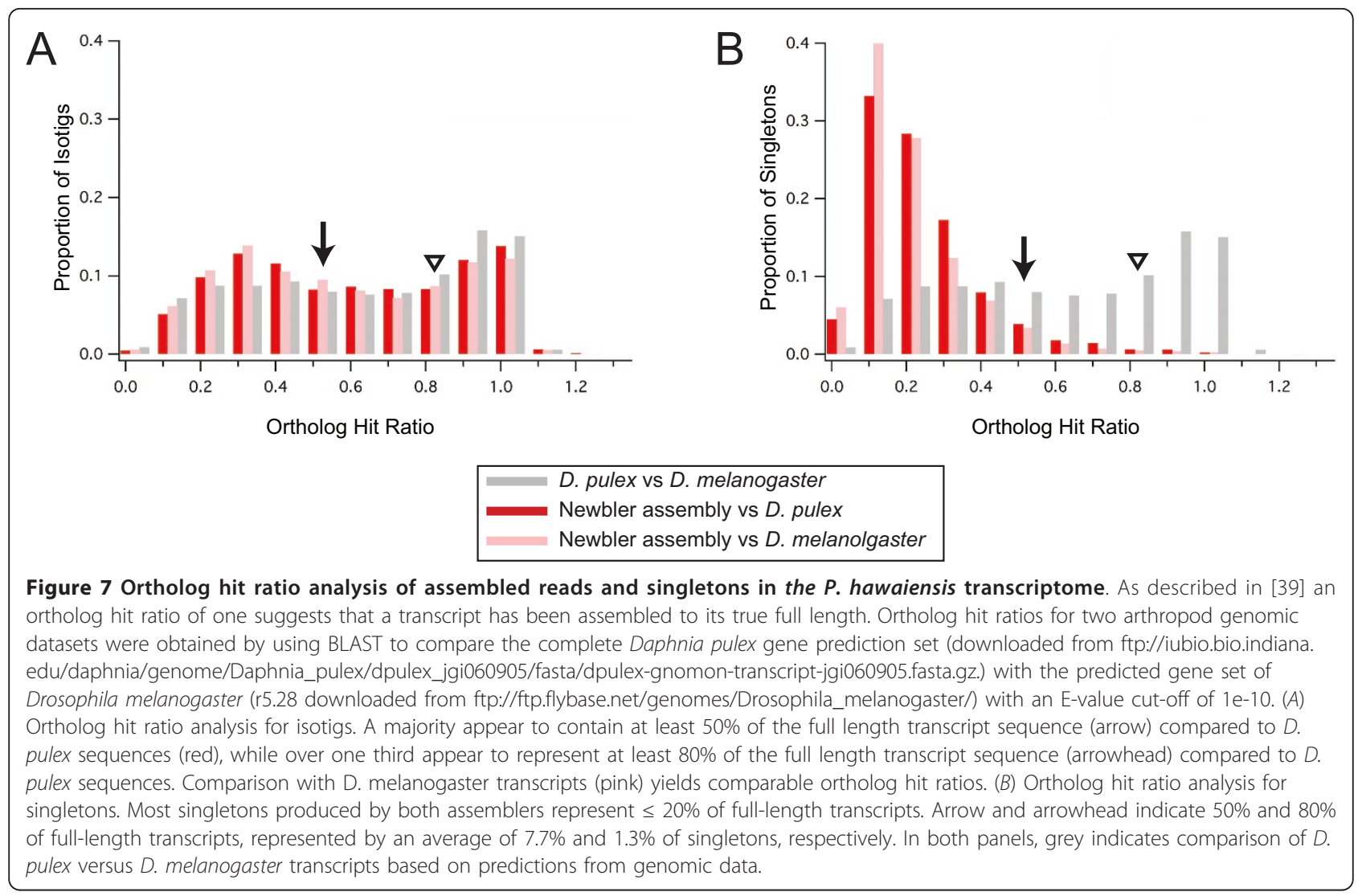

\section{Annotation of signaling pathway genes}

Future functional genetic studies in P. hawaiensis will likely focus on elucidating the function of highly conserved metazoan genes in this amphipod. We therefore annotated the transcriptome for the presence of genes belonging to major conserved animal signaling pathways [58]. Using the KEGG pathways as a guideline [59], we searched for $P$. hawaiensis homologues of these genes using BLAST using an E-value cutoff of $1 \mathrm{e}-10$. In most cases, the D. melanogaster homologue of a gene was used as a query, but for some searches, homologues from other organisms were used as queries (Additional File 9). For the Notch, TGF $\beta$, Wnt, Hedgehog, JAK/STAT and MAPK pathways, considering pathway members known from all animals, we identified likely $P$. hawaiensis homologues of an average of $52.8 \%$ $(103 / 195)$ of pathway genes (Figure 8$)$. If we consider only those pathway members with known $D$. melanogaster homologues $(n=138)$, this proportion is an average of $74.6 \%$. The proportion of genes found for each pathway ranged from $58.8 \%$ (MAPK pathway) to $93.8 \%$ (JAK/STAT pathway). Several genes of interest were found among the singletons. Although singletons are sometimes discarded before transcriptome annotation [see for example 23], our data suggest that even these unassembled reads can be a rich source of gene discovery. The transcriptome sequences for these genes ranged in length from $276 \mathrm{bp}$ (presenilin,
Notch pathway) to 4,882 bp ( $C K 2$, Wnt pathway), and the majority are at least $500 \mathrm{bp}$ long, making them immediately useful for in situ hybridization, RNAi-mediated gene knockdown, and RACE [60] in the case that longer or flanking genomic sequences are required for specific applications. Interestingly, for several signaling pathway members without a $D$. melanogaster homologue, we found $P$. hawaiensis homologues (Figure 8), suggesting that in some respects, amphipod signaling pathways may bear greater resemblance to vertebrate pathways than fruit flies.

\section{Annotation of developmental and gametogenesis genes}

Given the tractability of $P$. hawaiensis as a developmental model, we sought to make this transcriptome of maximal immediate use to the amphipod and broader developmental biology and biomedical research communities. We therefore undertook manual annotation of over 450 genes involved in embryonic patterning, developmental pathways (Additional File 10) and gametogenesis in both males and females (Additional File 11). We used lists of genes known to function in these processes in Drosophila as a starting point http://www.sdbonline.org/fly/aimain/1aahome.htm, and identified over 200 likely P. hawaiensis homologues of these genes. As we observed for our annotation of signaling pathway genes, the majority of matching transcriptome 


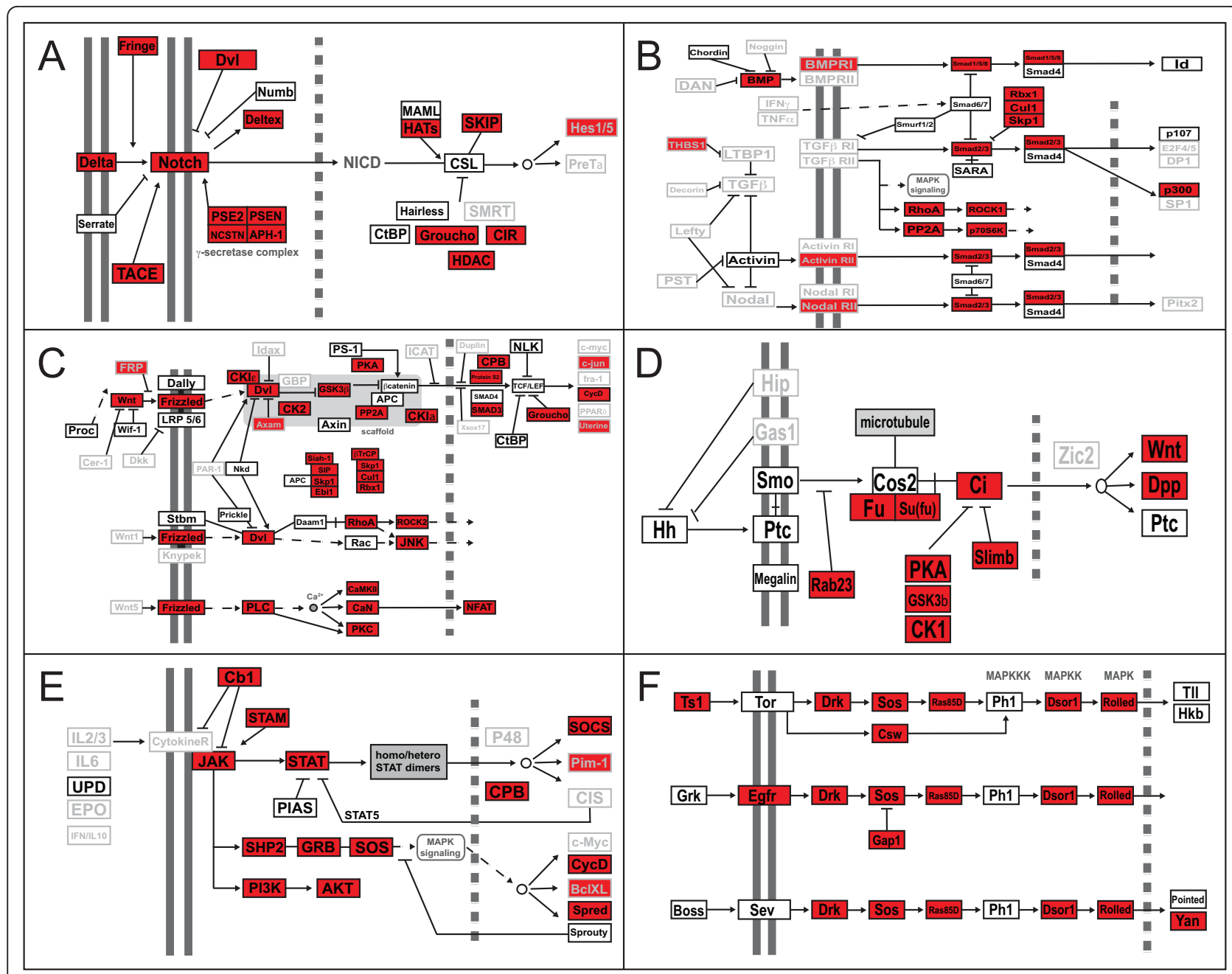

Figure 8 Conserved metazoan signal transduction pathway components identified in the $P$. hawaiensis transcriptome. These results were generated by using BLAST to compare sequences from known homologues against the full Newbler v2.5 assembly (see Additional File 9 and text for details), with genes identified marked in red. Genes outlined in grey with grey typeface indicate genes without $D$. melanogaster homologues. Pathway schematics modified from KEGG pathway model images http://www.genome.jp/kegg/kegg1.html. (A) Notch pathway. (B) TGF $\beta$ pathway. (C) Wnt pathway. (D) Hedgehog pathway. (E) Janus Kinase (JAK)-signal transducer and activator of transcription (STAT) pathway. (F) Mitogen-activated protein Kinase (MAPK) pathway.

reads are of sufficient length to allow immediate use in gene expression and function studies.

Several of the genes contained multiple hits in our transcriptome, including a large number of singletons. For some genes, these multiple hits included consecutively numbered isotigs with very similar lengths. We examined a subset of these genes to determine whether or not these apparently highly similar isotigs were in fact different from each other. We found that such sequences usually differed from each other at a small number of nucleotide positions, either because of low sequencing quality in one of the component reads, or because of SNPs or small indels (Additional File 12). Although annotation of SNPs in this transcriptome is beyond the scope of this study, we anticipate that the SNPs present in this transcriptome could serve as a useful tool for population-level variation studies in the future.

\section{Conclusions}

We have generated a maternal and embryonic transcriptome of the amphipod crustacean Parhyale hawaiensis using 454 Titanium pyrosequencing. To our knowledge, this represents the second largest crustacean genomic resource, behind the genome of the cladoceran D. pulex, and the largest de novo assembled pyrosequencing-based transcriptome to date. We confirmed the previously reported presence of trans-splicing in P. hawaiensis [47], but found that the presence of these trans-spliced 
transcripts did not greatly increase the complexity of the transcriptome or impede assembly. The $P$. hawaiensis transcriptome appears to be enriched for a particular class of $\mathrm{C} 2 \mathrm{H} 2 \mathrm{Zn}$ finger-coding transcripts, which share high similarity with several Branchiostoma floridae transcripts, and is also reported to be an enriched sequence class in the genome of the pea aphid Acyrthosiphon pisum [48].

We annotated the de novo transcriptome for a large number of developmentally relevant genes, including major conserved metazoan signaling pathways. We found that even after assembly of over 3 million reads, gene discovery continued to increase, suggesting that the extremely large genome size of this amphipod may reflect high gene numbers, high transcript complexity, or both. These data should both facilitate future developmental and evolutionary studies using this emerging model crustacean species, and contribute to future work in crustacean comparative genomics.

The bulk of existing arthropod genomic resources are for insects, while the sister group to the insects, the crustaceans, remains relatively unexplored. The large genome size and low relative similarity to existing annotated arthropod genomes may be challenges to potential future efforts to sequence the $P$. hawaiensis genome. However, high throughput short read sequencing technologies such as Illumina or SOLiD [61], combined with the transcriptome described here, should make such projects tractable.

Finally, this dataset should assist phylogenomic approaches to resolution of controversies in crustacean phylogenetic relationships, including the relationship between crustaceans and hexapods. D. pulex belongs to the Branchiopoda, and while some phylogenetic analyses place this group closest to the hexapods [62-65], others suggest that the Malacostraca (the group including $P$. hawaiensis and most edible crustaceans such as shrimp, lobster and crab) are sister to the hexapods [4,9-11,66]. Yet others suggest that Branchiopoda may be the most derived group within a monophyletic clade containing all crustaceans except for Malacostraca, Remipedia and Cephalocarida [67]. The largest phylogenomic assessment of this problem to date places both Branchiopoda and Malacostraca in a monophyletic clade that is sister to a (Hexapoda + Remipedia + Cephalocarida) clade [6] Moreover, Daphnia species do not branch basally within the Branchiopoda, and indeed are placed in the most derived branchiopod clade by most phylogenetic analyses $[6,68,69]$. These competing hypotheses emphasize the importance of a reliable comparative genomic framework within which to consider findings from new crustacean models. In order to place future comparative, ecological, environmental and biomedical studies using crustacean models in an appropriate phylogenetic context, additional crustacean genomic resources will be necessary.

\section{Methods}

\section{Animal culture}

The $P$. hawaiensis (Figure 1A, B) specimens sequenced in this study were from an inbred, non-backcrossed, non-isogenic laboratory culture originally obtained from Ernst Wimmer in 2002; the Wimmer culture in turn was obtained from a laboratory culture from Nipam H. Patel that was established with animals from the John G. Shedd Aquarium (Chicago, IL) as previously described [55]. The animals were maintained in the laboratory in artificial seawater (Instant Ocean, specific gravity 1.018-1.022), and fed a mixture of raw carrots, TetraAlgae Vegetable Enhanced Crisps, TetraMin Tropical Flakes, and Hikari Wheat Germ Pellets. All cultures were maintained under a 12:12 light/dark cycle at $28^{\circ} \mathrm{C}$.

\section{cDNA Synthesis}

471 mixed-stage embryos (Additional File 1; total weight $52.7 \mathrm{mg}$ ) representing the entirety of embryogenesis (Figure 1D) were shock frozen in liquid nitrogen and stored at $-80^{\circ} \mathrm{C}$. 30 ovaries (comprising eight small ovaries from young females and 22 late ovaries containing mature oocytes from older females; Figure 1C) were dissected from females in Trizol, flash frozen in Trizol, and stored at $-80^{\circ} \mathrm{C}$. Total RNA was isolated separately from ovaries (Figure 1C) and from mixed stages of embryogenesis (Figure 1E), and a pool was created of $1.5 \mu \mathrm{g}$ of total RNA from each sample for use as a template for first strand cDNA synthesis ( $3 \mu \mathrm{g}$ total). cDNA was synthesized following a protocol developed specifically for 454 Titanium sequencing of cDNA [22], with the exception that none of the cDNA was normalized in the present study. This protocol is based on the SMART cDNA library construction kit (Clontech, CA, USA), and includes a modified poly $(\mathrm{T})$ primer to enrich for mRNA, and a DNAse treatment step to remove possible genomic DNA contamination. Following first strand cDNA synthesis, primary amplification of the cDNA required thirteen PCR cycles to maximize yield while avoiding overcycling (monitored in real-time via qPCR [22]). Secondary amplification began to plateau after nine cycles. To obtain sufficient double-stranded cDNA for pyrosequencing sequencing $(\sim 5 \mu \mathrm{g})$ without overcycling, 26 reactions of $100 \mu \mathrm{g}$ each were run in parallel and subsequently copurified into $90 \mu \mathrm{l}$ of elution buffer using QIAquick PCR purification columns (Qiagen Inc).

\section{Titanium Pyrosequencing}

The samples were nebulized, adaptor-ligated, and pyrosequenced using the GS-FLX Titanium platform by the Institute for Genome Science and Policy DNA Sequencing Facility (Duke University). All of the raw reads generated 
in this study have been submitted to the NCBI Short Read Archive (Study Accession Number: SRA021010).

\section{Sequence Assembly}

Raw reads were assembled using the cDNA assembly algorithm (the "-cdna" flag) of Newbler v2.5. An adaptor-trimming step was included in the assembly (the "-vt" flag). Screening adaptors used are available at http://extavourlab.com/protocols/ExtavourLab_454_Adapters.fasta). A vector-screening step (the "-vs" flag) was performed using a FASTA version of the Univec database ftp://ftp.ncbi.nih. gov/pub/UniVec/. Due to the long average length of the raw 454 reads, the minimum overlapping length (the "-ml" flag) was set to the default value of 40 base pairs. To accommodate the possible existence of SNPs and pyrosequencing errors, the minimum identity between sequences (the "-mi" flag) was set to require 95\% identity between aligned sequences. Trimmed singleton reads were specifically produced by Newbler (the "-trim" flag). A maximum isogroup size of 10 isotigs was specified (the "-it" flag) to prevent Newbler from constructing an isogroup using an overly large quantity of short contigs. The resulting assembled reads and unassembled singletons were used for all subsequent analyses.

\section{Sequence Annotation}

Sequences were first mapped against the nr (all nonredundant GenBank CDS translations+PDB+SwissProt + PIR+PRF) peptide sequence database [70, downloaded from ftp://ftp.ncbi.nih.gov/blast/db/on April 27, 2010] using BLASTX. Unless otherwise specified, all BLAST searches were conducted using BLAST v2.2.24+ [71] with an E-value cutoff of 1e-10.

To annotate Gene Ontology (GO) terms [72] and their parents associated with the top 50 BLAST hits for each sequence, we used Blast2GO v1.2.7 [46].

Due to the sequence depth of this project, the transcripts were assembled with the raw 454 reads were not all full-length. If sequences represent different regions of the same transcript that are not assembled together due to insufficient overlap, they run the risk of being counted twice in our BLAST annotation. To address this problem, a custom Perl script was created in order to estimate the number of unique transcripts present in the $P$. hawaiensis transcriptome (called "UniqueBlast.pl" available at http://www.extavourlab.com/protocols/bio_tools/Perl_Transcriptome_Analysis_Scripts.zip). UniqueBlast.pl utilizes the results of BLASTX against $\mathbf{n r}$ for all assembly products with an E-value cutoff of 1e-10, to predict whether multiple assembly products are fragments of the same transcript. UniqueBlast.pl accomplishes this by comparing the HSP region of assembly products with the same top BLAST hit. Assembly products that have the same top BLAST hit but non-overlapping HSP regions are considered as fragments of the same transcript. Assembly products with the same top BLAST hits and overlapping HSP regions are considered as putative isoforms or paralogues of each other. Overlapping is defined as greater than 14 amino acids shared within the HSP, because the assembly parameters require a minimum of 40 matching nucleotides in order for two raw reads to be assembled together. UniqueBlast.pl then generates a list of assembly sequences (isotigs or contigs) showing the longest fragment of each transcript, as well as predicted isoforms and paralogues. This list of unique transcripts was then used for all subsequent analysis including GO analysis, ortholog hit ratio calculations, and phylogenetic distribution analysis of top BLAST hit organisms.

For analysis of the phylogenetic distribution of species of top unique matches to non-redundant assembly sequences, we used BLASTX to compare our non-redundant assembly products (isotigs + singletons, see Table 2) with nr using an E-value cutoff of 1e-10. We discarded redundant hits as described using "UniqueBlast.pl." For each gene in this list of unique BLAST hits, we recorded the species identity of the top BLAST hit sequence. We then tallied the total numbers for clades of interest, shown in Figure 4 and Additional File 5. When assessing the effect of Branchiostoma floridae $\mathrm{C} 2 \mathrm{H} 2 \mathrm{Zn}$ finger sequences on the annotation of the assemblies, we assessed top BLAST hits obtained with both the NCBI reference Sequence collection (RefSeq) and $\mathbf{n r}$, and obtained comparable results for both searches.

For comparison of sequences belonging to different GO terms between species, we used the Oncopeltus fasciatus data from a previously generated transcriptome [22] and a precomputed GO annotation of the D. melanogaster genome [73]. To obtain GO category data for Daphnia pulex, we used the transcripts predicted from the $D$. pulex genome (downloaded on 3 December 2010 from ftp://iubio. bio.indiana.edu/daphnia/genome/Daphnia_pulex/dpulex_jgi060905/fasta/dpulex-gnomon-transcript-jgi060905. fasta.gz).

To search for developmental genes of interest, we used TBLASTN with protein queries being the full length Drosophila melanogaster homologue of the gene of interest. For genes that yielded no hits when the $D$. melanogaster homologue was used as a query, homologues from other animals were used as queries. In cases where proteins possessed domains that were also shared by other genes from a different gene family (e.g. abstrakt: zinc finger domain), the TBLASTN search was performed by masking the relevant domain in the query sequence. Finally, in cases of genes with small, diagnostic conserved domains within an otherwise poorly conserved sequence, the diagnostic domains were used as the query (e.g. groucho). An E-value cutoff of e-10 was used for all BLAST searches, except for those searches 
with masked domains or specific protein domains, in which case the E-value cutoff was e-5. For all developmental genes found, species identity and domain details of the query used are indicated in the legends to Additional Files 9, 10 and 11.

\section{Removal of reads from trans-spliced sequences}

To determine how the presence of trans-spliced sequences affected our assembly with Newbler, we used the $P$. hawaiensis splice leader sequences [as per Figure 1 of 47] as an "adapter" sequence (the "-vs" flag) in the trimming step performed by Newbler prior to assembly. This resulted in removal or trimming of all raw reads containing the splice leader sequence. The remaining reads were assembled with Newbler v2.5 and compared using BLAST against $\mathbf{n r}$ as described for the complete assembly.

\section{Removal of $\mathrm{C} 2 \mathrm{H} 2$ Zinc finger-containing sequences}

Because we suspected that specific characteristics of $P$. hawaiensis $\mathrm{C} 2 \mathrm{H} 2 \mathrm{Zn}$-finger containing proteins might be responsible for a high incidence of BLAST hits to sequences of the lancelet Branchiostoma floridae, we performed a new assembly after removing a subset of reads in the following way: we scanned all reads for the presence of $\mathrm{C} 2 \mathrm{H} 2 \mathrm{Zn}$ finger-encoding sequences using the least stringent $\mathrm{C} 2 \mathrm{H} 2$ motif defined in [50], which is $\mathrm{X}_{2}-\mathrm{C}-\mathrm{X}_{1,2,4,5}{ }^{-} \mathrm{C}$ $\mathrm{X}_{12}-\mathrm{H}-\mathrm{X}_{3-6^{-}}(\mathrm{H}, \mathrm{C})$. We used this least stringent criterion rather than the most stringent criterion defined by Böhm and colleagues[50], in order to capture the largest number of reads containing these motifs. We reasoned that even "C2H2-like" domains might result in a Zn finger BLAST match, thus skewing the proportions of B. floridae hits. We used using a custom script ("C2H2.pl" available at http://www.extavourlab.com/protocols/bio_tools/Perl_Transcriptome_Analysis_Scripts.zip) and removed reads with matches. The remaining reads were assembled with Newbler v2.5, and the resulting assembly was scanned again for the presence of $\mathrm{C} 2 \mathrm{H} 2 \mathrm{Zn}$ finger-encoding sequences; assembled reads with hits were discarded. The remaining sequences were compared with $\mathbf{n r}$ using BLAST.

\section{Estimating sequencing depth and transcript completion}

To determine to what extent we had saturated gene discovery in the libraries we sequenced, we performed independent assemblies of ten progressively larger, randomly sampled subsets of the reads. The total number of genes in each sub-assembly was then identified via BLASTX against $\mathbf{n r}$. If multiple isotigs or contigs hit non-overlapping portions of the same top BLAST hit, only one of these sequences was counted. To calculate the ortholog hit ratio [39], we first used the script for generating a list of unique BLAST results described above ("UniqueBlast.pl"). We then used a custom ortholog hit ratio script ("OrthologHitRatio.pl" available at http://www.extavourlab.com/protocols/bio_tools/ Perl_Transcriptome_Analysis_Scripts.zip) to calculate the values used to create the graphs in Figure 6.

\section{Estimating extent of gene family expansion}

We first identified the $D$. pulex transcripts belonging to the duplicated gene families described by Colbourne and colleagues et al (Figure S31 in [2]) based on the KEGG enzyme code. We recorded the NCBI Gnomon transcript prediction ID of each $D$. pulex transcript that was listed with the chosen KEGG enzyme codes. We then mapped all of the $P$. hawaiensis isotigs against the $D$. pulex Gnomon-predicted transcriptome using TBLASTN. All $P$. hawaiensis isotigs with a top BLAST hit matching any recorded $D$. pulex NCBI Gnomon transcript prediction ID were identified. If the $P$. hawaiensis isotigs identified in this way belonged to the same isogroup, only a single isotig from that isogroup was counted. Using this method, we counted the putative number of $P$. hawaiensis paralogues from a chosen gene family.

[The sequence data from this study have been submitted to GenBank under study accession number SRA021010. Custom scripts generated are available at http://www.extavourlab.com/protocols/bio_tools/Python $\% 20$ Transcriptome\%20Analysis\%20Tools.tar.gz and http://www.extavourlab.com/protocols/bio_tools/Perl_Transcriptome_Analysis_Scripts.zip. Assembly results are available at http://www.extavourlab.com/resources/ index.html and at http://www.bio.miami.edu/wbrowne/ BrowneLab2/Community_Resources.html.]

\section{Additional material}

\footnotetext{
Additional file 1: Embryonic stages pooled for creation of the $P$. hawaiensis transcriptome. Staging as per [55].

Additional file 2: Comparison of read lengths from Newbler v2.5 de novo assembly of the $\boldsymbol{P}$. hawaiensis transcriptome. (A) Distribution of read lengths after assembly with Newbler v2.5 (red). (B) Distribution of read lengths of the shortest assembled reads and raw reads. The assembly yielded assembled reads of over $\sim 4000 \mathrm{bp}$.

Additional file 3: Distribution of average coverage (reads/bp) within contigs produced by Newbler v2.5 de novo assembly of the $P$.

hawaiensis transcriptome. The coverage within contigs is calculated by dividing the total number of base pairs contained in the reads used to construct a contig by the length of that contig.

Additional file 4: Analysis of the effect of trans-splicing transcripts on de novo transcriptome assembly. Assembly of all trimmed sequences compared to assembly of sequences lacking the trans-splicing leader sequences [47]. Number of BLAST hits reflects a search against the $\mathbf{n r}$ database with an E-value cut-off value of 1e-10.

Additional file 5: Phylogenetic distribution of species of top unique BLAST hit for Newbler v2.5 assembly of the $P$. hawaiensis transcriptome. Of the unique BLAST hits to all non-redundant assembly products (isotigs + singletons), $90 \%$ were from species belonging to the clades shown. Over $50 \%$ of these top BLAST hits are from arthropod species. The large number (12.2\%) of top BLAST hits in the complete
} 
assembly to sequences from Branchiostoma floridae is due to the high similarity of $\mathrm{C} 2 \mathrm{H} 2$ zinc finger domain-containing sequences with a particular linker sequence (TGEKP) that is also highly represented in the genome of the aphid Acyrthosiphon pisum [48]. Red: values after removal of reads and sequences containing this domain. Phylogenetic tree modified from $[63,74,75]$. Hits from the following most abundant species are represented: D. mojavensis, D. willistoni, D. ananassae, D. grimshawi, $D$. pseudoobscura pseudoobscura, Aedes aegypti, Anopheles gambiae, Culex quinquefasciatus (Flies \& Mosquitoes), Bombyx mori (Moth), Tribolium castaneum (Beetle), Harpegnathos saltator, Camponotus floridanus, Apis mellifera, Nasonia vitripennnis (Bee, Wasp \& Ants), Acyrthosiphon pisum (Aphid), Pediculus humanus corporis (Louse), Ixodes scapularis (Tick), Penaeus monodon, Lepeophtheirus salmonis, Litopenaeus vannamei (Crustaceans), Caenorhabditis remanei (Nematode), Gallus gallus, Taeniopygia guttata (Birds), Rattus norvegicus, Mus musculus, Monodelphis domestica (Mammals), Xenopus laevis, X. tropicalis (Amphibian), Danio rerio, Tetraodon nigroviridis (Fish), Branchiostoma floridae (Amphioxus), Ciona intestinalis (Sea Squirt), Saccoglossus kowalevskii (Acorn Worm), Strongylocentrotus purpuratus (Sea Urchin), Trichoplax adherens (Trichoplax), Hydra magnipapillata, Nematostella vectensis (Cnidarians), Perkinsus marinus (Dinoflagellate).

Additional file 6: Sequences with strong similarity to Daphnia pulex gene sequences identified in the de novo $P$. hawaiensis transcriptome. Because the $D$. pulex genome and $\mathbf{n r}$ are databases of inevitably different sizes, E-values shown here are for information only and are not strictly comparable. See text for additional details.

\section{Additional file 7: Presence of existing P. hawaiensis GenBank} accessions in the de novo transcriptome. Sequences of $P$. hawaiensis developmental genes from GenBank were used as a query to BLAST the de novo transcriptome. Most genes with hits had several matches in the transcriptome, among both assembled reads and singletons.

Additional file 8: The $P$. hawaiensis transcriptome adds sequence data to GenBank accession number HM191476, the P. hawaiensis prospero homologue. Extended contig for Ph-prospero, comprising the complete mRNA GenBank accession (top, light grey), one isotigs and one contig from the Newbler assembly of the transcriptome (dark grey). The isotig provides an additional $445 \mathrm{bp}$ of $3^{\prime}$ UTR sequence and $116 \mathrm{bp}$ of 5' UTR sequence (black) to the GenBank sequence. Comparison with the GenBank sequence shows that isotig24415 and singleton

GAP9EXG06HFGHB belong to the same contig.

Additional file 9: Selected signaling pathway genes identified in the P. hawaiensis transcriptome. Hit ID indicates if gene hits were found assembled reads (A) or singletons (S). Sequence length (range) indicates the shortest and longest $\mathrm{A}$ or $\mathrm{S}$ hit sequences for each gene. These results are shown graphically in Figure 7. Groups of hits of a given colour indicate transcriptome sequences that mapped to the same overlapping region of the BLAST target; hits of different colours indicate

transcriptome sequences that map to different, non-overlapping regions of the BLAST target. Query organisms: $D m=D$. melanogaster; $\mathrm{Dr}=$ Danio rerio; $X t=$ Xenopus tropicalis. Query sequence details: 1. Kinase domain was masked. 2. FERM domain used as query. 3. Amino acids 500-833 (DI/ Ser domain) used as query. 4. Amino acids 1-250 (groucho/TLE domain) used as query. 5. Kinase domain masked; amino acids 420-1390 used as query. 6. Kinase domain masked; amino acids 175-372 used as query. 7. Kinase domain masked; amino acids 150-516 used as query. 8. Kinase domain masked; amino acids 1-100 used as query. 9. Kinase domain masked; amino acids 1-890 used as query. Asterisks indicate genes that appear elsewhere in the same table (in a different pathway).

Additional file 10: Selected developmental process genes identified in the $P$. hawaiensis transcriptome. Hit ID indicates if gene hits were found assembled reads (A) or singletons (S). Sequence length (range) indicates the shortest and longest $\mathrm{A}$ or $\mathrm{S}$ hit sequences for each gene. Groups of hits of a given colour indicate transcriptome sequences that mapped to the same overlapping region of the BLAST target; hits of different colours indicate transcriptome sequences that map to different, non-overlapping regions of the BLAST target. Query organism was $D$. melanogaster for all cases. Boldface indicates genes also present in other tables (Additional Files 9, 11); asterisks indicate genes that appear elsewhere in the same table (in a different functional category).
Additional file 11: Selected genes involved in gametogenesis identified in the $P$. hawaiensis transcriptome. Hit ID indicates if gene hits were found assembled reads (A) or singletons (S). Sequence length (range) indicates the shortest and longest $\mathrm{A}$ or $\mathrm{S}$ hit sequences for each gene. Groups of hits of a given colour indicate transcriptome sequences that mapped to the same overlapping region of the BLAST target; hits of different colours indicate transcriptome sequences that map to different, non-overlapping regions of the BLAST target. Query organism was D. melanogaster for all cases. Query sequence details: 1. S/T kinase domain was masked. 2. Dead box/Zn finger domains were masked. 3. HLH domain was masked 4. Peptidase C14 domain was masked. 5. Kinase domain masked; amino acids 175-372 used as query. 6. BTB domain used as query. 7. Kinase domain masked; amino acids 1-890 used as query. Boldface indicates genes also present in other tables (Additional Files 9, 10); asterisks indicate that genes are also present elsewhere (in a different functional category) in the same table.

Additional file 12: Representative of consecutively numbered isotigs with highly similar lengths. An example of two isotigs which both have Cyclin D as their top BLAST hit (see Additional File 9), differ in length by only two nucleotides, and have highly similar sequences. Isotig07129 is 4,279 bp long; isotig07130 is 4,277 bp long. Only a portion of the sequence of each isotig is shown. Nucleotide positions differing between the two are indicated in black (likely to be SNPS), white (deletions) or grey (apparent sequence difference may be due to poor quality sequence (lower case letters) at this position).

\section{Acknowledgements}

Thanks to Friedemann Linsler for assistance with embryo collection, Lisa Bukovnik for her administration of the sequencing, Ana Conesa and Stefan Götz for valuable comments regarding use of the Blast2GO tool, and the Extavour lab for discussions of the results. This work was partially supported by NSF grant IOS-0817678, Ellison Medical Foundation New Scholar Award AG-NS-07010-10, Harvard Stem Cell Institute Seed Grant SG-0057-10-00 (VZ) and a Milton Fund Grant (FA) to CE, an NSF Predoctoral Fellowship to BEC, and funding from the University of Miami College of Arts and Sciences to WEB.

\section{Author details}

'Department of Organismic and Evolutionary Biology, Harvard University, 16 Divinity Avenue, Cambridge, MA 02138, USA. ${ }^{2}$ Department of Biology, University of Miami, 234 Cox Science Center, 1301 Memorial Drive, Coral Gables, FL 33146, USA.

\section{Authors' contributions}

VZ performed experiments, helped design data analysis and analyzed data. KEV performed experiments and analyzed data. BEC helped design research, performed experiments, collected and analyzed data. FA performed ovary dissections and embryo collections. WEB helped design the research, performed experiments, analyzed data and obtained funding for the research. CGE proposed the idea for the research, helped design the research and analyze the data, wrote the manuscript with input from all authors, and obtained funding for the research. All authors read and approved the final manuscript.

Received: 10 August 2011 Accepted: 25 November 2011 Published: 25 November 2011

\section{References}

1. Brusca GJ, Brusca RC: Invertebrates. 2 edition. Sunderland, MA: Sinauer Associates Inc.; 2003.

2. Colbourne JK, Pfrender ME, Gilbert D, Thomas WK, Tucker A, Oakley TH, Tokishita S, Aerts A, Arnold GJ, Basu MK, et al: The ecoresponsive genome of Daphnia pulex. Science 2011, 331:555-561.

3. Mallatt JM, Garey JR, Shultz JW: Ecdysozoan phylogeny and Bayesian inference: first use of nearly complete $28 \mathrm{~S}$ and $18 \mathrm{~S}$ rRNA gene sequences to classify the arthropods and their kin. Mol Phylogenet Evol 2004, 31:178-191. 
4. Friedrich M, Tautz D: Ribosomal DNA phylogeny of the major extant arthropod classes and the evolution of myriapods. Nature 1995, 376:165-167.

5. Boore JL, Lavrov DV, Brown WM: Gene translocation links insects and crustaceans. Nature 1998, 392:667-668.

6. Regier JC, Shultz JW, Zwick A, Hussey A, Ball B, Wetzer R, Martin JW, Cunningham CW: Arthropod relationships revealed by phylogenomic analysis of nuclear protein-coding sequences. Nature 2010, 463:1079-1083.

7. Giribet G, Edgecombe GD, Wheeler WC: Arthropod phylogeny based on eight molecular loci and morphology. Nature 2001, 413:157-161.

8. Richter S, Moller OS, Wirkner CW: Advances in Crustacean Phylogenetics. Arthropod Systematics and Phylogeny 2009, 67:275-286.

9. Hwang UW, Friedrich M, Tautz D, Park CJ, Kim W: Mitochondrial protein phylogeny joins myriapods with chelicerates. Nature 2001, 413:154-157.

10. Harzsch S: The phylogenetic significance of crustacean optic neuropils and chiasmata: a re-examination. J Comp Neurol 2002, 453:10-21

11. Sinakevitch I, Douglass JK, Scholtz G, Loesel R, Strausfeld NJ: Conserved and convergent organization in the optic lobes of insects and isopods, with reference to other crustacean taxa. J Comp Neurol 2003, 467:150-172

12. Wilson K, Cahill V, Ballment E, Benzie J: The complete sequence of the mitochondrial genome of the crustacean Penaeus monodon: are malacostracan crustaceans more closely related to insects than to branchiopods? Mol Biol Evol 2000, 17:863-874.

13. Stillman JH, Colbourne JK, Lee CE, Patel NH, Phillips MR, Towle DW, Eads BD, Gelembuik GW, Henry RP, Johnson EA, et al: Recent advances in crustacean genomics. Integr Comp Biol 2008, 48:852-868

14. Vega EDI, O'Leary NA, Robalino J, Peck ME, Bartlett TC, Richards M, Hikima S, Browdy CL, Warr GW, Chapman RW, Gross PS: Litopenaeus vannamei Gene Discovery Project. Book Litopenaeus vannamei Gene Discovery Project. 2008 edition. City: NCBl; 2008.

15. Alcivar-Warren A, Delaney M, Meehan-Meola D, Alcivar MN, Warren WB: Expressed sequence tags from cadmium-exposed postlarvae stage 42 of cultured, specific pathogen-free Pacific whiteleg shrimp, Litopenaeus vannamei. Book Expressed sequence tags from cadmium-exposed postlarvae stage 42 of cultured, specific pathogen-free Pacific whiteleg shrimp, Litopenaeus vannamei. 2009 edition. City: NCBl; 2009.

16. Muller IC, Stocco PH, Marques MRF: Differentially expressed genes in gills of farmed shrimp Litopenaeus vannamei infected with White Spot Synfrome Virus. Book Differentially expressed genes in gills of farmed shrimp Litopenaeus vannamei infected with White Spot Synfrome Virus. 2010 edition. City: NCBI; 2010.

17. Nilsen $\mathrm{F}$, Malde $\mathrm{K}$, Kongshaug $\mathrm{H}$ : Functional genomics in sea lice. Book Functional genomics in sea lice. 2010 edition. City: NCBI; 2010.

18. Tagmount $A$, Wang $M$, Lindquist $E$, Tanaka $Y$, Teranishi $K S$, Sunagawa $S$, Wong $\mathrm{M}$, Stillman JH: The porcelain crab transcriptome and PCAD, the porcelain crab microarray and sequence database. PLOS ONE 2010, 5 : e9327.

19. Towle DW, Smith CM, Beale KM: Expressed Sequence Tags in a normalized cDNA library prepared from multiple tissues of adult intermolt and postmolt American lobster, Homarus americanus. Book Expressed Sequence Tags in a normalized cDNA library prepared from multiple tissues of adult intermolt and postmolt American lobster, Homarus americanus. 2007 edition. City: NCBI; 2007.

20. Verslycke T, Tarrant AM, Stegeman JJ, McDowell J: Expressed sequence tags associated with asymptomatic and shell-diseased lobsters. Book Expressed sequence tags associated with asymptomatic and shell-diseased lobsters. 2009 edition. City: NCBl; 2009

21. Stepanyan R, Day K, Urban J, Hardin DL, Shetty RS, Derby CD, Ache BW, McClintock TS: Gene expression and specificity in the mature zone of the lobster olfactory organ. Physiol Genomics 2006, 25:224-233.

22. Ewen-Campen B, Shaner N, Panfilio KA, Suzuki Y, Roth S, Extavour CG: The maternal and embryonic transcriptome of the milkweed bug Oncopeltus fasciatus. BMC genomics 2011, 12:61.

23. Clark MS, Thorne MA, Toullec JY, Meng Y, Guan le L, Peck LS, Moore S: Antarctic krill 454 pyrosequencing reveals chaperone and stress transcriptome. PLOS ONE 2011, 6:e15919.

24. Dana JD: Crustacea. In US Exploring Edition during the years 1838-1842. Volume XIII. Edited by: Wilkes C. C. Sherman; 1852.

25. Rehm EJ, Hannibal RL, Chaw RC, Vargas-Vila MA, Patel NH: The Crustacean Parhyale hawaiensis, A New Model for Arthropod Development. In
Emerging Model Organisms: A Laboratory Manual. Volume 1. Cold Spring Harbor: CSHL Press; 2008:373-404.

26. Gerberding M, Browne WE, Patel NH: Cell lineage analysis of the amphipod crustacean Parhyale hawaiensis reveals an early restriction of cell fates. Development 2002, 129:5789-5801.

27. Alwes F, Hinchen B, Extavour CG: Patterns of cell lineage, movement, and migration from germ layer specification to gastrulation in the amphipod crustacean Parhyale hawaiensis. Dev Biol 2011.

28. Price AL, Modrell MS, Hannibal RL, Patel NH: Mesoderm and ectoderm lineages in the crustacean Parhyale hawaiensis display intra-germ layer compensation. Dev Biol 2010, 341:256-266.

29. Pavlopoulos A, Averof M: Establishing genetic transformation for comparative developmental studies in the crustacean Parhyale hawaiensis. Proc Natl Acad Sci USA 2005, 102:7888-7893.

30. Pavlopoulos A, Kontarakis Z, Liubicich DM, Serano JM, Akam M, Patel NH, Averof M: Probing the evolution of appendage specialization by Hox gene misexpression in an emerging model crustacean. Proc Natl Acad Sci USA 2009, 106:13897-13902.

31. Kontarakis Z, Pavlopoulos A, Kiupakis A, Konstantinides N, Douris V, Averof $\mathrm{M}$ : A versatile strategy for gene trapping and trap conversion in emerging model organisms. Development 2011

32. Vargas-Vila MA, Hannibal RL, Parchem RJ, Liu PZ, Patel NH: A prominent requirement for single-minded and the ventral midline in patterning the dorsoventral axis of the crustacean Parhyale hawaiensis. Development 2010, 137:3469-3476.

33. Özhan-Kizil G, Havemann J, Gerberding M: Germ cells in the crustacean Parhyale hawaiensis depend on Vasa protein for their maintenance but not for their formation. Dev Biol 2009, 327:320-239.

34. Liubicich DM, Serano JM, Pavlopoulos A, Kontarakis Z, Protas ME, Kwan E, Chatterjee S, Tran KD, Averof M, Patel NH: Knockdown of Parhyale Ultrabithorax recapitulates evolutionary changes in crustacean appendage morphology. Proc Natl Acad Sci USA 2009, 106:13892-13896.

35. Parchem RJ, Poulin F, Stuart AB, Amemiya CT, Patel NH: BAC library for the amphipod crustacean, Parhyale hawaiensis. Genomics 2010, 95:261-267.

36. Venter JC, Adams MD, Myers EW, Li PW, Mural RJ, Sutton GG, Smith HO, Yandell $\mathrm{M}$, Evans CA, Holt RA, et al: The sequence of the human genome. Science 2001, 291:1304-1351.

37. McPherson JD, Marra M, Hillier L, Waterston RH, Chinwalla A, Wallis J, Sekhon M, Wylie K, Mardis ER, Wilson RK, et al: A physical map of the human genome. Nature 2001, 409:934-941.

38. Sander K: Specification of the basic body pattern in insect embryogenesis. Advances in Insect Physiology 1976, 12:125-238.

39. O'Neil ST, Dzurisin JDK, Carmichael RD, Lobo NF, Emrich SJ, Hellmann JJ: Population-level transcriptome sequencing of nonmodel organisms Erynnis propertius and Papilio zelicaon. BMC genomics 2010, 11:310

40. Parchman TL, Geist KS, Grahnen JA, Benkman CW, Buerkle CA: Transcriptome sequencing in an ecologically important tree species: assembly, annotation, and marker discovery. BMC genomics 2010, 11:180

41. Roeding F, Borner J, Kube M, Klages S, Reinhardt R, Burmester T: A 454 sequencing approach for large scale phylogenomic analysis of the common emperor scorpion (Pandinus imperator). Mol Phylogenet Evol 2009, 53:826-834.

42. Novaes E, Drost DR, Farmerie WG, Pappas GJ Jr, Grattapaglia D, Sederoff RR, Kirst M: High-throughput gene and SNP discovery in Eucalyptus grandis, an uncharacterized genome. BMC genomics 2008, 9:312.

43. Vera JC, Wheat CW, Fescemyer HW, Frilander MJ, Crawford DL, Hanski I, Marden JH: Rapid transcriptome characterization for a nonmodel organism using 454 pyrosequencing. Mol Ecol 2008, 17:1636-1647.

44. Hahn DA, Ragland GJ, Shoemaker DD, Denlinger DL: Gene discovery using massively parallel pyrosequencing to develop ESTs for the flesh fly Sarcophaga crassipalpis. BMC genomics 2009, 10:234

45. Meyer E, Aglyamova GV, Wang S, Buchanan-Carter J, Abrego D, Colbourne JK, Willis BL, Matz MV: Sequencing and de novo analysis of a coral larval transcriptome using 454 GSFIx. BMC genomics 2009, 10:219.

46. Conesa A, Götz S, Garcia-Gomez JM, Terol J, Talon M, Robles M: Blast2GO: a universal tool for annotation, visualization and analysis in functional genomics research. Bioinformatics 2005, 21:3674-3676.

47. Douris V, Telford MJ, Averof M: Evidence for multiple independent origins of trans-splicing in Metazoa. Mol Biol Evol 2010, 27:684-693.

48. Shigenobu S, Bickel RD, Brisson JA, Butts T, Chang C-C, Christiaens O, Davis GK, Duncan EJ, Ferrier DEK, Iga $M$, et al: Comprehensive survey of 
developmental genes in the pea aphid, Acyrthosiphon pisum: frequent lineage-specific duplications and losses of developmental genes. Insect Mol Biol 2010, 19(Suppl 2):47-62.

49. Holland LZ, Albalat R, Azumi K, Benito-Gutiérrez E, Blow MJ, BronnerFraser M, Brunet F, Butts T, Candiani S, Dishaw LJ, et al: The amphioxus genome illuminates vertebrate origins and cephalochordate biology. Genome Res 2008, 18:1100-1111.

50. Böhm S, Frishman D, Mewes HW: Variations of the $\mathrm{C} 2 \mathrm{H} 2$ zinc finger motif in the yeast genome and classification of yeast zinc finger proteins. Nucleic Acids Res 1997, 25:2464-2469.

51. Jin L, Kryukov K, Clemente JC, Komiyama T, Suzuki Y, Imanishi T, Ikeo K, Gojobori T: The evolutionary relationship between gene duplication and alternative splicing. Gene 2008, 427:19-31.

52. Browne WW, Schmid BGM, Wimmer EA, Martindale MQ: Expression of otd orthologs in the amphipod crustacean, Parhyale hawaiensis. Development Genes and Evolution 2006, 216:581-595.

53. Browne WE: The embryonic development of the amphipod crustacean Parhyale hawaiensis. PhD University of Chicago, Molecular Genetics and Cell Biology; 2003.

54. O'Day KE: Notch signaling and segmentation in Parhyale hawaiensis. PhD University of California, Berkeley, Molecular and Cell Biology; 2006.

55. Browne WE, Price AL, Gerberding M, Patel NH: Stages of embryonic development in the amphipod crustacean, Parhyale hawaiensis. Genesis 2005, 42:124-149.

56. Price AL: Gastrulation and Mesodem Development in Parhyale hawaiensis. University of Chicago, Committee on Developmental Biology; 2005.

57. Price AL, Patel NH: Investigating divergent mechanisms of mesoderm development in arthropods: the expression of Ph-twist and Ph-mef2 in Parhyale hawaiensis. Journal of Experimental Zoology Part B: Molecular Development and Evolution 2008, 310:24-40.

58. Pires-daSilva A, Sommer RJ: The evolution of signalling pathways in animal development. Nat Rev Genet 2003, 4:39-49.

59. Kanehisa M, Goto S, Furumichi M, Tanabe M, Hirakawa M: KEGG for representation and analysis of molecular networks involving diseases and drugs. Nucleic Acids Res 2010, 38:D355-360.

60. Frohman MA: On beyond classic RACE (rapid amplification of CDNA ends). PCR Methods App/ 1994, 4:540-58.

61. Morozova O, Marra MA: Applications of next-generation sequencing technologies in functional genomics. Genomics 2008, 92:255-264.

62. Mallatt J, Giribet G: Further use of nearly complete $28 \mathrm{~S}$ and $18 \mathrm{~S}$ rRNA genes to classify Ecdysozoa: 37 more arthropods and a kinorhynch. Mol Phylogenet Evol 2006, 40:772-794.

63. Dunn CW, Hejnol A, Matus DQ, Pang K, Browne WE, Smith SA, Seaver E, Rouse GW, Obst M, Edgecombe GD, et al: Broad phylogenomic sampling improves resolution of the animal tree of life. Nature 2008, 452:745-749.

64. Hejnol A, Obst M, Stamatakis A, Ott M, Rouse GW, Edgecombe GD, Martinez P, Baguna J, Bailly X, Jondelius U, et al: Assessing the root of bilaterian animals with scalable phylogenomic methods. Proc $R$ Soc Lond B Biol Sci 2009, 276:4261-4270.

65. Gaunt MW, Miles MA: An insect molecular clock dates the origin of the insects and accords with palaeontological and biogeographic landmarks. Mol Biol Evol 2002, 19:748-761.

66. Fanenbruck M, Harzsch S, Wagele JW: The brain of the Remipedia (Crustacea) and an alternative hypothesis on their phylogenetic relationships. Proc Natl Acad Sci USA 2004, 101:3868-3873.

67. Koenemann S, Jenner RA, Hoenemann M, Stemme T, von Reumont BM: Arthropod phylogeny revisited, with a focus on crustacean relationships. Arthropod Structure \& Development 2010, 39:88-110.

68. Olesen J: Phylogeny of Branchiopoda (Crustacea) - Character Evolution and Constribution of Uniquely Preserved Fossils. Arthropod Systematics and Phylogeny 2009, 67:3-39.

69. Stenderup JT, Olesen J, Glenner H: Molecular phylogeny of the Branchiopoda (Crustacea)-multiple approaches suggest a 'diplostracan' ancestry of the Notostraca. Mol Phylogenet Evol 2006, 41:182-194.

70. Pruitt KD, Tatusova T, Maglott DR: NCBI reference sequences (RefSeq): a curated non-redundant sequence database of genomes, transcripts and proteins. Nucleic Acids Res 2007, 35:D61-65.

71. Camacho C, Coulouris G, Avagyan V, Ma N, Papadopoulos J, Bealer K, Madden TL: BLAST+: architecture and applications. BMC Bioinformatics 2009, 10:421.
72. Ashburner M, Ball CA, Blake JA, Botstein D, Butler H, Cherry JM, Davis AP, Dolinski K, Dwight SS, Eppig JT, et al: Gene ontology: tool for the unification of biology. The Gene Ontology Consortium. Nat Genet 2000, 25:25-29.

73. B2G-FAR: A Species Centered GO Annotation Repository. [http://bioinfo. cipf.es/b2gfar/showspecies?species=7227].

74. Ishiwata K, Sasaki G, Ogawa J, Miyata T, Su Z-H: Phylogenetic relationships among insect orders based on three nuclear protein-coding gene sequences. Mol Phylogenet Evol 2010, 158:169-180.

75. Wheeler WC, Whiting M, Wheeler QD, Carpenter JM: The phylogeny of the extant hexapod orders. Cladistics 2001, 17:113-169.

doi:10.1186/1471-2164-12-581

Cite this article as: Zeng et al:: De novo assembly and characterization of a maternal and developmental transcriptome for the emerging model crustacean Parhyale hawaiensis. BMC Genomics 2011 12:581.

\section{Submit your next manuscript to BioMed Central and take full advantage of:}

- Convenient online submission

- Thorough peer review

- No space constraints or color figure charges

- Immediate publication on acceptance

- Inclusion in PubMed, CAS, Scopus and Google Scholar

- Research which is freely available for redistribution

Submit your manuscript at www.biomedcentral.com/submit
Biomed Central 